\title{
TRANSIENT JETS IN THE SYMBIOTIC PROTOTYPE Z ANDROMEDAE
}

\author{
A. Skopal, T. Pribulla ${ }^{1}$ and J. Budaj ${ }^{2}$ \\ Astronomical Institute, Slovak Academy of Sciences, SK-05960 Tatranská Lomnica, Slovakia \\ A. A. Vittone and L. Errico \\ INAF Osservatorio Astronomico di Capodimonte, via Moiariello 16, I-80131 Napoli, Italy \\ M. Wolf \\ Astronomical Institute, Charles University Prague, CZ-180 00 Praha 8, V Holešovičkách 2, Czech \\ Republic \\ M. Otsuka \\ Okayama Astrophysical Observatory, NAOJ, Kamogata, Okayama 719-0232, Japan \\ and \\ M. Chrastina and Z. Mikulášek \\ Institute of Theoretical Physics and Astrophysics, Masaryk University Brno, Kotlářská 2, \\ CZ-611 37 Brno, Czech Republic
}

\begin{abstract}
We present the development of the collimated bipolar jets from the symbiotic prototype Z And that appeared and disappeared during its 2006 outburst. We monitored the outburst with the optical high-resolution spectroscopy and multicolor $U B V R_{\mathrm{C}}$ photometry. In 2006 July Z And reached its historical maximum at $U \sim 8$.0. After $\sim 1$ mag decline in mid-August, it kept its brightness at a high level of $U \sim 9$ up to 2007 January. During this period, rapid photometric variations with $\Delta m \sim 0.06$ mag on the timescale of hours developed. Simultaneously, high-velocity satellite components appeared on both sides of the $\mathrm{H} \alpha$ and $\mathrm{H} \beta$ emission line profiles. Their presence was transient, being detected to the end of 2006. They were launched asymmetrically with the red/blue velocity ratio of $1.2-1.3$. From about mid-August onward they became symmetric at $\sim \pm 1200 \mathrm{~km} \mathrm{~s}^{-1}$, reducing the velocity to $\sim \pm 1100 \mathrm{~km} \mathrm{~s}^{-1}$ at their disappearance. Spectral properties of these satellite emissions indicated the ejection of
\end{abstract}

\footnotetext{
${ }^{1}$ Visiting Astronomer: David Dunlap Observatory

${ }^{2} 2003-2006$, visiting astronomer at the Department of Astronomy, Pennsylvania State University
} 
bipolar jets collimated within an average opening angle of $6^{\circ} .1$. If the jets were expelled at the escape velocity then the mass of the accreting white dwarf is $M_{\mathrm{WD}} \sim 0.64 M_{\odot}$. We estimated the average outflow rate via jets to $\dot{M}_{\text {jet }} \sim 2 \times 10^{-6}\left(R_{\text {jet }} / 1 \mathrm{AU}\right)^{1 / 2} M_{\odot} \mathrm{yr}^{-1}$, during their August-September maximum, which corresponds to the emitting mass in jets, $M_{\text {jet }}^{\text {em }} \sim 6 \times 10^{-10}\left(R_{\text {jet }} / 1 \mathrm{AU}\right)^{3 / 2} M_{\odot}$. During their lifetime, the jets released the total mass of $M_{\text {jet }}^{\text {total }} \approx 7.4 \times 10^{-7} M_{\odot}$. Evolution in the rapid photometric variability and asymmetric ejection of jets around the optical maximum can be explained by a disruption of the inner parts of the disk caused by radiation-induced warping of the disk.

Subject headings: stars: binaries: symbiotic - stars: individual: Z And - ISM: jets and outflows

\section{Introduction}

Collimated jets from astrophysical objects represent very exciting events. They have been detected from various kinds of objects. A common element in all jet-producing systems is the central accreting star, which implies that their accretion disks play an important role in the formation of this type of mass outflow (e.g. Livio 1997, 2004, and references therein).

Symbiotic stars are long-period interacting binaries (orbital periods are in the range of years), in which a white dwarf (WD) accretes material from the wind of a cool giant. This process generates a very hot and luminous source of radiation $\left(T_{\mathrm{h}} \approx 10^{5} \mathrm{~K}, L_{\mathrm{h}} \approx 10^{2}-10^{4} L_{\odot}\right)$ and accumulates a certain amount of material onto the WD's surface that enlarges its effective radius to about $0.1 R_{\odot}$ during their quiescent phases. During active phases a large, geometrically and optically thick circumstellar disk with radius of a few $R_{\odot}$ develops around the accretor (Skopal 2005, Figs. 26 and 27). Therefore, the symbiotic stars are good candidates for producing high-velocity outflows in the form of collimated jets, mainly during outbursts. However, signatures of collimated outflows were indicated for only 10 from roughly 200 known symbiotics (Brocksopp et al. 2004 ): seven by resolving their spatial structure with the radio and X-ray imaging (e.g. Tavlor et al. 1986; Crocker et al. 2001; Gallowav \& Sokoloski 2004) and three by the optical spectroscopy (e.g. Tomov et al. 2000; Schmid et al. 2001). Nevertheless, jets in symbiotic binaries are investigated very intensively from X-ray to radio wavelengths to understand better the physical process driving the collimated high-velocity mass outflow from accreting white dwarfs (e.g. Sokoloski et al. 2004; Stute \& Camenzind 2005; Karovska et al. 2007; Kellogg et al. 2007; Stute \& Sahai 2007).

$\mathrm{Z}$ And is considered a prototype symbiotic star. Its recent activity started from 2000 autumn and reached the optical maxima in 2000 December, 2004 September and 2006 July (Fig. 1). During the previous well observed large eruptions $(1985,2000)$, signatures of high-velocity outflows were detected through the broadening of emission lines, P-Cygni type of profiles and the extended wings of hydrogen lines (e.g. Fernández-Castro et al. 1995; Tomov et al. 2003, 2008; Sokoloski et al. 
2006; Skopal et al. 2006; Skopal 2006), but never in the form of collimated bipolar jets. The only signature for a nonsymmetric outflow from $\mathrm{Z}$ And was indicated by the $5 \mathrm{GHz}$ radio map from 2001 September, which showed a transient jet-like extension (Brocksopp et al. 2004). However, no counterpart in the optical spectrum could be recognized (Burmeister \& Leedjärv 2007). Recently, during the maximum of the 2006 outburst, Skopal \& Pribulla (2006) discovered spectral signatures of bipolar jets from Z And. Their evidence in the optical spectra was confirmed by Burmeister \& Leediärv (2007) and Tomov et al. (2007).

In this contribution we investigate evolution of the jet features from their first appearance in 2006 July to their disappearance at the end of 2006, determine their physical parameters, and discuss the disk-jet connection during the outburst.

\section{Observations and data reduction}

Our observations of Z And during its 2006 outburst were carried out at different observatories.

(i) At the McDonald Observatory by the 9.2-m Hobby-Eberly telescope (Ramsey et al. 1998, HET in Table 1) just prior to the rise of the major 2006 outburst. Observations were performed using the echelle high dispersion spectrograph (Tull 1998) with the 316 g5936 cross-disperser, $3^{\prime \prime}$ fiber, and 2 CCDs $(4096 \times 4100)$ with $2 \times 1$ binning. Data were reduced using the standard IRAF procedures, which involved bias, bad pixels, flatfield, scattered light, continuum, and cosmic rays corrections. Wavelength calibration was done using Th-Ar lamp and has an error of about $0.1 \mathrm{~km} \mathrm{~s}^{-1}$. Observations were recorded in 71 orders which cover the spectral regions 4060-5880, 6010-7760 with the resolution of about $\mathrm{R}=60000$ and $\mathrm{S} / \mathrm{N} \sim 200$ in the continuum. For the purpose of this paper we use only regions containing $\mathrm{H} \alpha, \mathrm{H} \beta$, He II 4686, He I 4713 and the Raman-scattered O VI 6825 line.

(ii) At the David Dunlap Observatory, University of Toronto (DDO) the high-resolution spectroscopy was performed by the single dispersion slit spectrograph equipped with a Jobin Yovon Horiba CCD detector $(2048 \times 512$ pixels of $13.5 \mu \mathrm{m}$ size; thinned back illuminated chip) mounted at the Cassegrain focus of the 1.88-m telescope. The resolution power was 12000 and 8000 around the $\mathrm{H} \alpha$ and He II 4686- $\mathrm{H} \beta$ region, respectively. Basic treatment of the spectra was done using the IRAF-package software. During each night two very different exposures were applied to obtain a well defined $\mathrm{H} \alpha$ profile and the continuum. Telluric sharp absorptions in the $\mathrm{H} \alpha$ region were eliminated with the aid of simultaneously observed standard star 50 Boo.

(iii) At the Asiago Astrophysical Observatory (Asiago). Here the high-dispersion spectroscopy was secured by the REOSC echelle spectrograph equipped with a AIMO E2VCCD47-10 back illuminated CCD detector $(1100 \times 1100$ pixels of $13 \mu \mathrm{m}$ size $)$ mounted at the Cassegrain focus of the 1.82-m telescope at Mt. Ekar. The resolution power with the $200 \mu \mathrm{m}$ width slit was approximately 25000 and 17000 at the $\mathrm{H} \alpha$ and He II $4686-\mathrm{H} \beta$ region, respectively. The spectroscopic data were processed with the MIDAS software package and a software developed at the Astronomical 

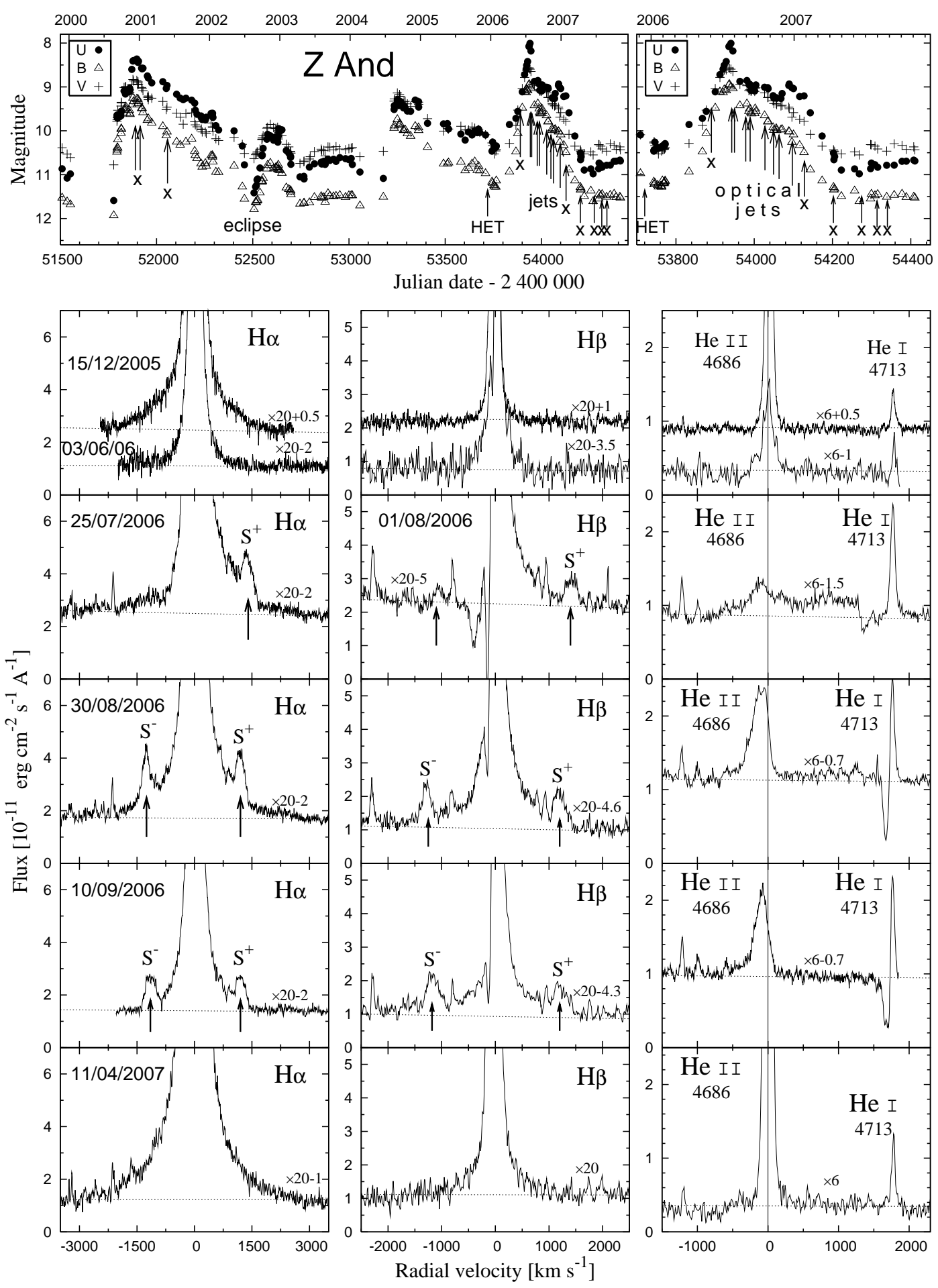

Fig. 1. - Top panels show the $U, B, V$ light curves (LCs) of $\mathrm{Z}$ And covering its two major eruptions that peaked in 2000 December and 2006 July. Arrows indicate times of spectroscopic observations. Absence of jets is denoted by " $x$ ". The lower block of panels displays spectral regions around $\mathrm{H} \alpha, \mathrm{H} \beta$, and He II $4686 \AA$ during the 2006 outburst. The jet features are denoted by $\mathrm{S}^{-}$and $\mathrm{S}^{+}$and arrows. Dotted lines represent the continuum. 
Table 1: Log of spectroscopic observations

\begin{tabular}{|c|c|c|c|c|}
\hline $\begin{array}{c}\text { Date } \\
(\mathrm{dd} / \mathrm{mm} / \text { уууу })\end{array}$ & $\begin{array}{c}\text { Julian Date } \\
\text { JD } 24 \ldots\end{array}$ & $\begin{array}{l}\text { Region } \\
(\mathrm{nm})\end{array}$ & $\begin{array}{l}T_{\exp } \\
(\min )\end{array}$ & Obs. \\
\hline $15 / 12 / 2005$ & 53720.127 & $406-776$ & $3 \times 10$ & HET \\
\hline \multirow[t]{3}{*}{$04 / 06 / 2006$} & 53890.528 & $462-472$ & 30 & Asiago \\
\hline & 53890.551 & $482-492$ & 30 & Asiago \\
\hline & 53890.575 & $652-666$ & 30 & Asiago \\
\hline $25 / 07 / 2006$ & 53942.809 & $642-671$ & 5 & DDO \\
\hline $01 / 08 / 2006$ & 53949.837 & $462-492$ & 15 & DDO \\
\hline \multirow[t]{2}{*}{$30 / 08 / 2006$} & 53978.688 & $462-492$ & 20 & DDO \\
\hline & 53978.706 & $642-671$ & 10 & DDO \\
\hline \multirow[t]{3}{*}{$10 / 09 / 2006$} & 53989.602 & $462-472$ & 30 & Asiago \\
\hline & 53989.626 & $482-492$ & 30 & Asiago \\
\hline & 53989.650 & $652-666$ & 30 & Asiago \\
\hline $18 / 10 / 2006$ & 54027.387 & $641-692$ & 33 & Ondřejov \\
\hline $09 / 11 / 2006$ & 54049.418 & $641-692$ & 50 & Ondřejov \\
\hline $23 / 11 / 2006$ & 54063.428 & $641-692$ & 50 & Ondřejov \\
\hline \multirow[t]{2}{*}{$27 / 12 / 2006$} & 54096.905 & $575-690$ & 20 & $\mathrm{OAO}$ \\
\hline & 54096.919 & $575-690$ & 20 & $\mathrm{OAO}$ \\
\hline \multirow{3}{*}{$30 / 01 / 2007$} & 54131.250 & $652-666$ & 5 & Asiago \\
\hline & 54131.263 & $652-666$ & 30 & Asiago \\
\hline & 54131.286 & $672-686$ & 30 & Asiago \\
\hline $11 / 04 / 2007$ & 54202.402 & $642-671$ & 5.5 & $\mathrm{DDO}$ \\
\hline $22 / 06 / 2007$ & 54274.697 & $642-671$ & 6.0 & $\mathrm{DDO}$ \\
\hline $31 / 07 / 2007$ & 54313.790 & $642-671$ & 3.0 & DDO \\
\hline $27 / 08 / 2007$ & 54340.485 & $652-686$ & 30 & Asiago \\
\hline
\end{tabular}


Observatory of Capodimonte in Napoli.

(iv) At the Ondřejov Observatory (Ondřejov) the high-resolution spectroscopy was performed by using the coude single-dispersion slit spectrograph of 2-m reflector and the BROR CCD camera with the SITe-005 $800 \times 2030$ pixels chip. The resolution power at the $\mathrm{H} \alpha$ region was 10000 . Standard initial reduction of CCD spectra (bias subtraction, flat-fielding and wavelength calibration) was carried out using modified MIDAS and IRAF packages. Final processing of the data was done with the aid of the SPEFO-package software developed at the Ondřejov Observatory (Horn et al. 1996; Š́koda 1996).

(v) At the Okayama Astrophysical Observatory (OAO) the high-dispersion spectra were secured with the high-dispersion echelle spectrograph (Izumiura 1999) at the f/29 coudé focus of the 1.88-m telescope. The dimension of the CCD (EEV 42-80) was $2048 \times 4096$ pixels of $13.5 \mu \mathrm{m}^{2}$. The red cross-disperser was used. The spectral resolving power was 54000 around the $\mathrm{H} \alpha$ region. The reduction and analysis was performed with the IRAF-package software. Two similar exposures were co-added to obtain a better signal-to-noise ratio $(\mathrm{S} / \mathrm{N})$.

(vi) At the Skalnaté Pleso and Stará Lesná (pavilion G2) Observatories, classical photoelectric $U B V R_{\mathrm{C}}$ measurements were carried out by single-channel photometers mounted in the Cassegrain foci of 0.6-m reflectors (see Skopal et al. 2004, in detail). The star BD+474192 (SAO 53150, $V$ $=8.99, B-V=0.41, U-B=0.14, V-R_{\mathrm{C}}=0.10$; $\mathrm{C} 1$ in Fig. 3) was used as the standard star for both photoelectric and CCD observations. Fast CCD photometry was performed at Stará Lesná Observatory using the 0.5-m telescope (pavilion G1). The SBIG ST10 MXE CCD camera with the chip $2184 \times 1472$ pixels and the $U B V(R I)_{C}$ Johnson-Cousins filter set were mounted at the Newtonian focus. The size of the pixel is $6.8 \mu \mathrm{m}$ and the scale $0.56^{\prime \prime} /$ pixel, corresponding to the field of view (FOV) of a CCD frame of about $24 \times 16$ arcmin.

(vii) At the MonteBoo Observatory of the Masaryk University in Brno (Czech Republic), additional high-time-resolution optical photometry was obtained during two nights, 2006 September 6 and 2006 September 11. The observational data were carried out using SBIG ST-8 dual chip CCD camera and Kron-Cousins $B V(R I)_{C}$ filter set attached at the Newtonian focus of the $602 / 2780$ telescope. The CCD chip has $1534 \times 1020$ pixels with the size of $9 \mu \mathrm{m}$ and the scale $2.04^{\prime \prime} /$ pixel (FOV $17.1 \times 11.4$ arcmin). All CCD frames were dark-subtracted, flat-fielded and corrected for cosmic rays. Heliocentric correction was applied for all data points. CCD photometric data were processed with the MIDAS software package and a software C-MUNIPACK 1 , developed at the Masaryk University in Brno. Other details of the CCD photometric reduction were described by Parimucha \& Vaňko (2005). To verify the reality of Z And rapid variations during selected nights we measured additional comparison stars: C2 (GSC 03645-01592, $V=11.17, B-V=0.53$ ), C3 (GSC2.2 N012002338, $B \sim 12.3 \mathrm{mag}$ ) and C4 (SAO 53133, $V=9.17, B-V=1.36, U-B=$ 1.11). The best results were achieved using the standard $\mathrm{C} 1$, because it is very similar to $\mathrm{Z}$ And

\footnotetext{
${ }^{1}$ See the project homepage http://c-munipack.sourceforge.net/
} 


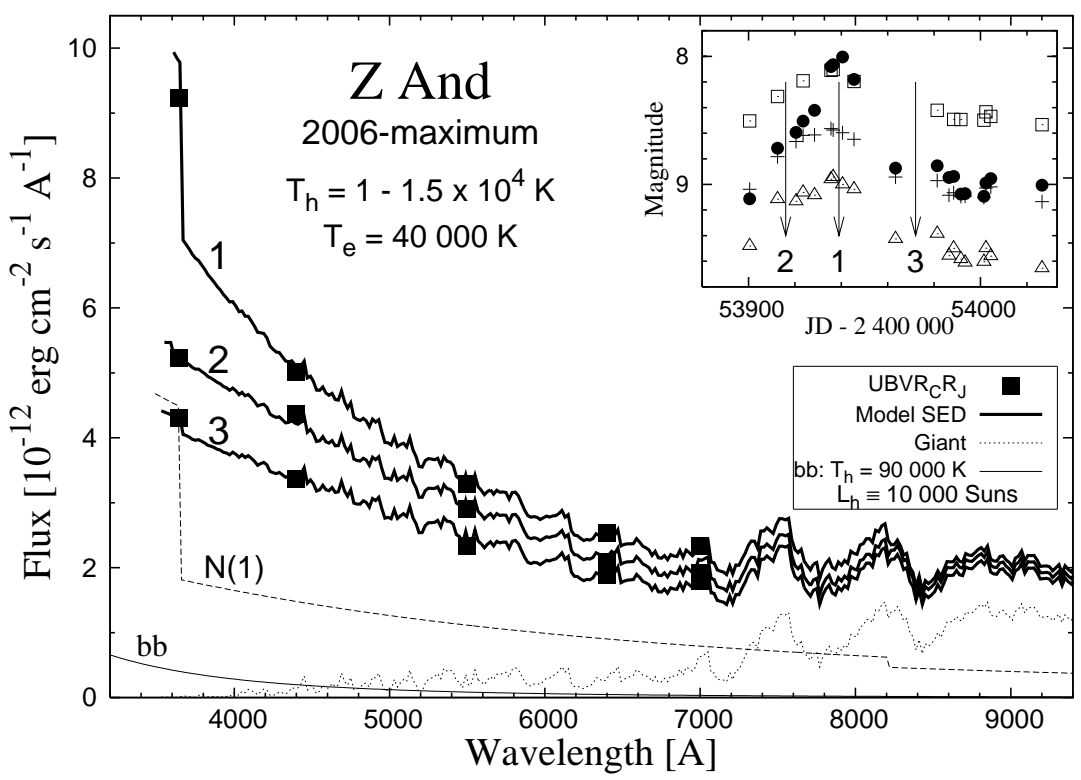

Fig. 2.- Observed (full boxes) and modeled (solid thick lines) SEDs at/around the 2006 maximum of $\mathrm{Z}$ And. Model for the red giant (dotted line) was adopted from Skopal (2005). Corresponding dates $(1,2,3)$ are shown on the $U B V R_{C} \operatorname{LCs}(\bullet, \triangle,+, \square)$ inserted at the right-up corner. At times 2 and 3 the nebular contribution was negligible, while at the maximum (1) it increased by a factor of $\sim 20$ (denoted by $\mathrm{N}(1)$ ). Radiation of a black body at the ionizing temperature of $90000 \mathrm{~K}(\mathrm{bb})$ is scaled to a maximum of the hot object luminosity of $\sim 10^{4} L_{\odot}$ (Sect. 3.3).

during outbursts in both the brightness and the spectral type (see Figs. 2 and 3). The errors of the Z And - C1 differences were mostly less than 0.01 mag.

The journal of spectroscopic observations is given in Table 1. A correction for heliocentric velocity was applied to all spectra. Arbitrary flux units were converted to absolute fluxes with the aid of the simultaneous $U B V R_{\mathrm{C}}$ photometry corrected for emission lines. The method was described by Skopal et al. (2006). Uncertainties of such a continuum calibration are of a few percent for the star's brightness around 9 mag in the $V R$ passbands (see Skopal 2007, in detail). Observations were dereddened with $E_{\mathrm{B}-\mathrm{V}}=0.30$ and resulting parameters were scaled to a distance of $1.5 \mathrm{kpc}$ (e.g. Mikolajewska \& Kenyon 1996).

\section{Analysis and results}

\subsection{Photometric evolution around the maximum}

The top-right panel of Fig. 1 shows the $U B V$ LCs of Z And covering its recent 2006 active phase. Prior to and after the peak July's magnitudes, the color index $U-V \sim 0$ (Figs. 1 and 2). In 
July, during the maximum, the index had became temporarily much bluer $(U-V \sim-0.55)$, which could be caused by a transient increase of the nebular emission. To understand better this behavior we modeled the $U B V R_{C} R_{J}$ flux points (Fig. 2) 2 using the method of disentangling the composite continuum as described by Skopal (2005). Our solutions correspond to a low temperature stellar source radiating at $T_{\mathrm{h}} \sim 10000-15000 \mathrm{~K}$ that dominates the optical spectrum. This suggests a significant contribution from a warm shell. At the maximum (the model 1 in Fig. 2), the SED requires a larger nebular contribution $\left(F_{\text {stellar }} / F_{\text {nebular }}=2.3\right.$ in $B$, emission measure $E M=9.4 \times$ $10^{60} \mathrm{~cm}^{-3}$ at $\left.T_{\mathrm{e}}=40000 \mathrm{~K}\right)$, than prior to and after it $\left(F_{\text {stellar }} / F_{\text {nebular }} \gtrsim 23\right.$ in $B, E M \sim 1.3-$ $\left.8.8 \times 10^{59} \mathrm{~cm}^{-3}\right)$. The range of temperatures and scaling factors in the models of the stellar source corresponds to its effective radius $R_{\mathrm{h}}^{\text {eff }}=12 \pm 4 R_{\odot}$. We note that the absence of ultraviolet data did not allow us to determine the model parameters precisely. Uncertainties in $T_{\mathrm{h}}$ and $T_{\mathrm{e}}$ are as high as 30 and $50 \%$, respectively.

The effect of the transient increase of the nebular emission at the maximum could be interpreted as a result of a mass ejection from the active object into the particle bounded nebula, because new emitters will convert the excess of ionizing photons there into the nebular radiation. The effect of brightening of symbiotic binary due to an increase in the mass-loss rate was originally suggested by Nussbaumer \& Vogel (1987).

In addition, we searched for a short-term photometric variability on the timescales of minutes to hours (Fig. 3). Just prior to and after the active stage we observed an irregular variation within $\Delta B \lesssim 0.02 \mathrm{mag}$ (top panels of Fig. 3; previously measured also by Gromadzki et al. 2006; Sokoloski \& Bildsten 1999), whereas around/after the maximum its amplitude increased by a factor of $\sim 3$, to $\Delta B \sim \Delta V \sim 0.06 \mathrm{mag}$ (bottom panels of Fig. 3; the first detection to date). Their source can be identified with the aid of the corresponding SED. The false $(1-1.5) \times 10^{4} \mathrm{~K}$ photosphere, which is associated with the disk, dominated the optical domain, while contributions from the giant and the nebula were negligible (Fig. 2). Therefore, the $\sim 0.06 \mathrm{mag}$ variations had to be produced by the disk. Also the large size of the disk $\left(R_{\mathrm{D}}>R_{\mathrm{h}}^{\mathrm{eff}} \approx 10 R_{\odot}\right)$ supports this interpretation - the timescale of the rapid photometric fluctuations is consistent with the dynamical time at the outer edge of the hot stellar source.

\subsection{Spectroscopic evolution}

Figures 1, 4 and 5 show evolution of the $\mathrm{H} \alpha, \mathrm{H} \beta$, He II 4686, He I $4713 \AA$, and the Ramanscattered O VI 1032 profiles throughout the whole 2006-07 outburst.

At the beginning of the 2006 active phase, prior to the initial rise (spectrum from 15/12/2005) as well as prior to the maximum (03/06/2006), the hydrogen and helium line profiles showed a simple

\footnotetext{
${ }^{2} R_{\mathrm{J}}$ fluxes were estimated from the measured $R_{\mathrm{C}}$ magnitudes, corrected for the $\mathrm{H} \alpha$ emission, using transformations of Bessell (1983) for the less red stars.
} 

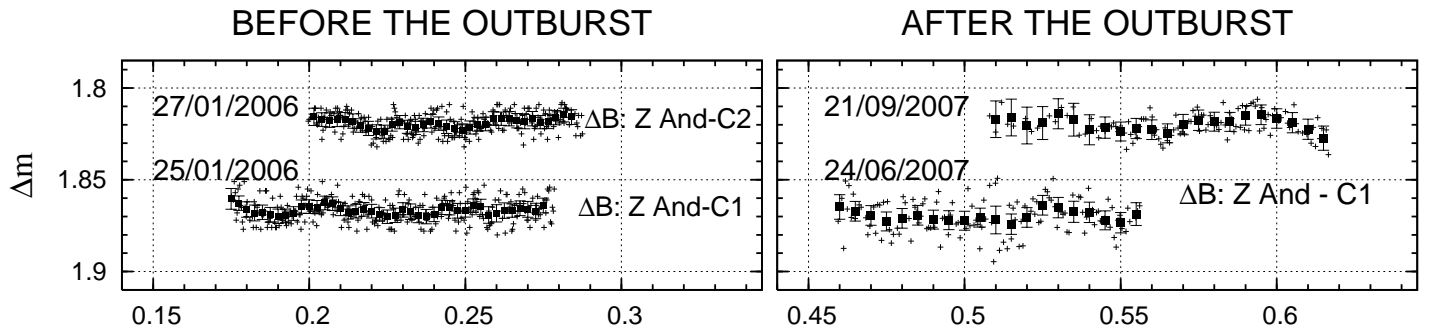

DURING THE OUTBURST
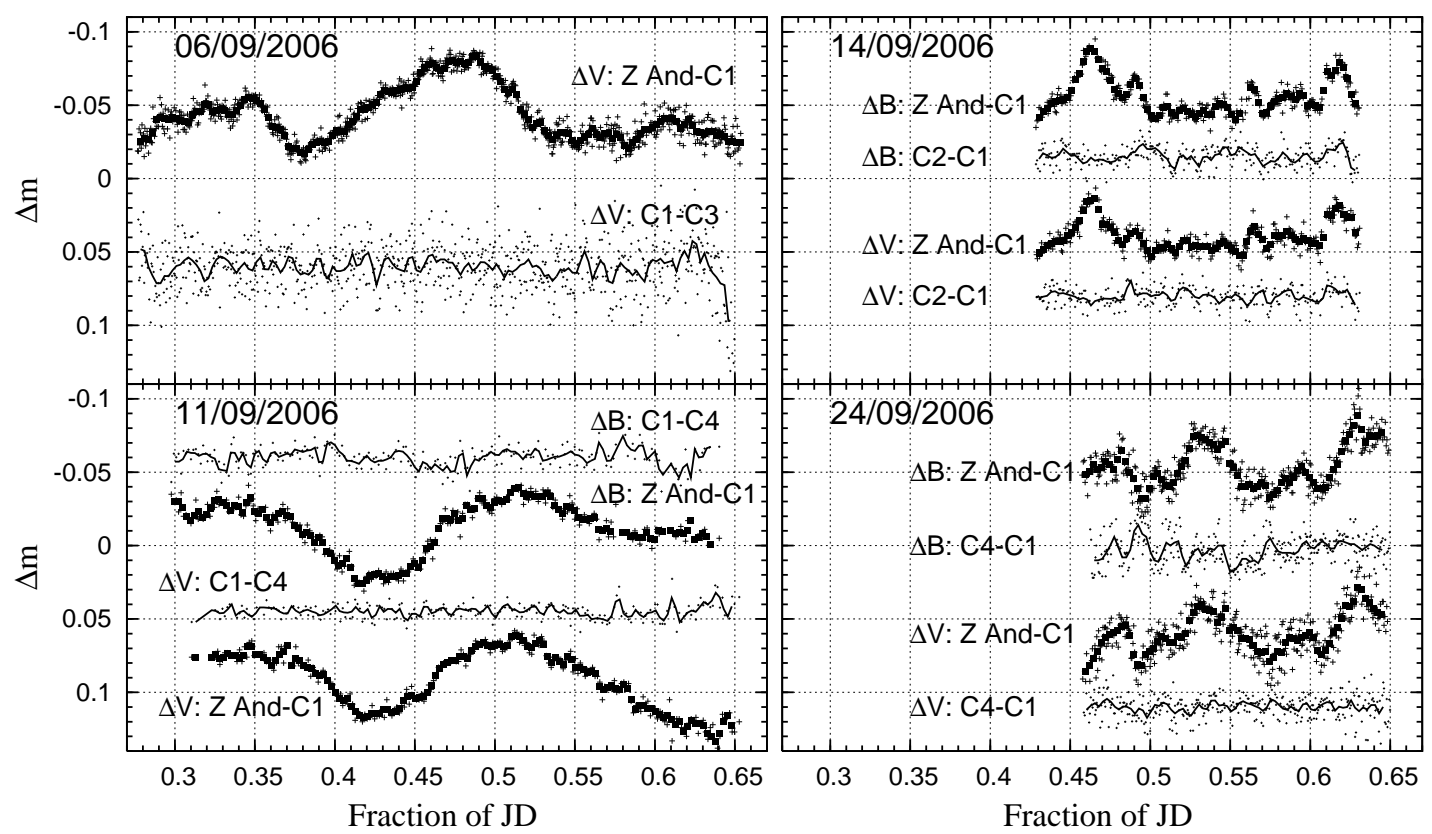

Fig. 3.- Short-term variability in the optical continuum prior to the 2006-outburst (top left), after it (top right) and around its maximum (lower panels). Crosses represent individual CCD measurements, while full squares their means within 3.6 - 7.2 minutes. Comparison stars are denoted by C1, C2, C3 and C4 (see Sect. 2). 
emission core. At these days, emission wings of $\mathrm{H} \alpha$ extended to $\sim \pm 1500$ and $\sim \pm 800 \mathrm{~km} \mathrm{~s}^{-1}$, respectively, while those of $\mathrm{H} \beta$ persisted within $\sim \pm 500 \mathrm{~km} \mathrm{~s}^{-1}$ on both spectra. The emission core of the He II 4686 line was placed symmetrically with respect to its reference wavelength (Fig. 1). Its strong flux in the 15/12/2005 spectrum, $\mathrm{F}$ (He II 4686) $=2.3 \times 10^{-11} \mathrm{erg} \mathrm{cm}^{-2} \mathrm{~s}^{-1}$, corresponded to the effective temperature of the ionizing source, $T_{\mathrm{h}}^{i . s .} \sim 140000 \mathrm{~K}(\mathrm{~F}(\mathrm{H} \beta)=5.5$ and $\mathrm{F}(\mathrm{He} \mathrm{I} 4471)=$ $0.16 \times 10^{-11} \mathrm{erg} \mathrm{cm}^{-2} \mathrm{~s}^{-1}$; Iijima 1981). In spite of a high temperature from nebular emission lines the Raman-scattered O VI 1032 line disappeared entirely (Fig. 5).

Our first spectrum from the maximum (25/07/2006) revealed a complex of strong absorptions in the $\mathrm{H} \alpha$ profile. One was extended to $\sim-1000 \mathrm{~km} \mathrm{~s}^{-1}$ and others cut the emission core at $\sim-220 \mathrm{~km} \mathrm{~s}^{-1}$ and $\sim-100 \mathrm{~km} \mathrm{~s}^{-1}$ (Fig. 4). In addition, a pronounced $\mathrm{S}^{+}$satellite component was present and located at $+1385 \mathrm{~km} \mathrm{~s}^{-1}$. One week later $(01 / 08 / 2006)$ strong absorptions at $\sim-400 \mathrm{~km} \mathrm{~s}^{-1}$ and $\sim-200 \mathrm{~km} \mathrm{~s}^{-1}$ appeared in the $\mathrm{H} \beta$ and He I $4713 \AA$ lines (Figs. 1 and 4 ). Two satellite components to the $\mathrm{H} \beta$ emission core were clearly recognizable and placed asymmetrically at $\sim-1100 \mathrm{~km} \mathrm{~s}^{-1}$ and $\sim+1400 \mathrm{~km} \mathrm{~s}^{-1}$. From August 30th we observed typical broad emission wings with the extension of about \pm 2000 and $\pm 1500 \mathrm{~km} \mathrm{~s}^{-1}$ in the $\mathrm{H} \alpha$ and $\mathrm{H} \beta$ profile, respectively. They were accompanied by pronounced bipolarly located satellite emissions. From October to December their fluxes were becoming fainter and at the end of 2007 January only some remnants in a form of faint spikes could be recognized (Fig. 4). We attribute these satellite components to bipolar jets. During the presence of jets, F(He II 4686) fluxes decreased significantly (F(He II 4686)/F(H $\beta) \sim 0.05$ ) and their profiles were shifted blueward (Fig. 1) as during the 2000-01 maximum (Skopal et al. 2006). The Raman line was not detectable (see also Burmeister \& Leediärv 2007). It became to be visible on our 27/12/2006 spectrum as a faint redward-shifted emission around $6838 \AA$ (Fig. 5).

Our spectra taken after the maximum, from 11/04/2007 to 27/08/2007, showed only a simple emission core of $\mathrm{H} \alpha$ and $\mathrm{H} \beta$ lines with decreasing extension of their emission wings (Fig. 4). He II 4686 line became stronger, being located again around its reference wavelength as prior to the outburst (Fig. 1). The Raman emission band strengthened significantly and was comparable in the profile with that from quiescent phase (Fig. 5).

\subsection{A disk-like structure of the hot object and its evolution}

Observed SEDs from the optical maximum require a very low temperature of the hot object, $T_{\mathrm{h}} \sim 1-1.5 \times 10^{4} \mathrm{~K}$ (Sect. 3.1). In contrast, a significantly higher temperature of the ionizing source, $T_{\mathrm{h}}^{i . s .} \sim 140000$ and $90000 \mathrm{~K}$, was derived from the hydrogen and helium emission lines prior to the outbursts (Sect 3.2) and during its maximum (Burmeister \& Leedjärv 2007), respectively. Thus the hot active object was characterized by the two-temperature type of spectrum with $T_{\mathrm{h}} \ll T_{\mathrm{h}}^{i . s}$. as during the 2000 maximum. This situation can be explained by a disk-like structure of the hot object viewed under a high inclination angle. Then the disk occults the hot ionizing source in the line of sight, while the nebula above/below the disk can easily be ionized (see Sect. 4.1. of Skopal et al. 2006, in detail). 


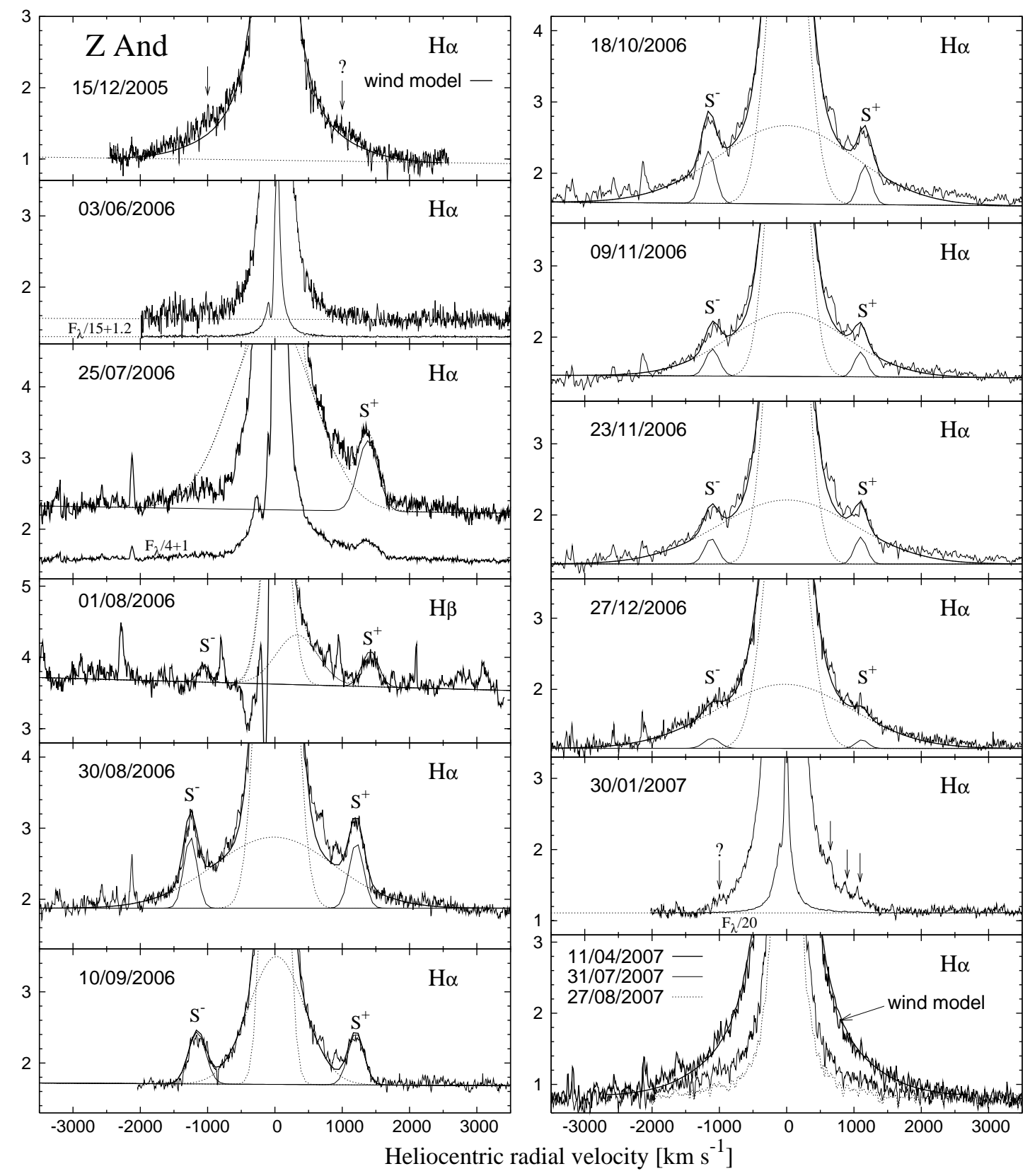

Fig. 4.- Evolution in the $\mathrm{H} \alpha(\mathrm{H} \beta)$ broad wings along the 2006 outburst. The jet emission components $\left(\mathrm{S}^{-}, \mathrm{S}^{+}\right.$, solid thin lines) developed during the optical maximum and were observable to the end of 2006 December. We isolated them from the total profile by fitting its central emission with two Gaussian functions (dotted curves). The wind model of the broad $\mathrm{H} \alpha$ wings (Sect. 3.9) is shown here for clarity only to the spectrum from 15/12/2005 and 11/04/2007. Corresponding parameters are in Table 2. Fluxes are in $10^{-12} \mathrm{erg} \mathrm{cm}^{-2} \mathrm{~s}^{-1} \AA^{-1}$. 
Another effect resulting from the disk-structured hot object is the blueward shift of the He II $4686 \AA$ profile that develops during the outbursts, while out of active phases it is placed symmetrically with respect to the reference wavelength (Fig. 1 here and Fig. 2 in Skopal et al. 2006). The size of the $\mathrm{He}^{++}$zone was probably very small at the maximum (note the very low flux of the He II 4686 line during the maximum, Sect. 3.2) and thus the disk blocked a fraction of its redward shifted emission in the direction of the observer for the orbital inclination of about $75^{\circ}$.

The disappearance of the Raman line during active phases at high $T_{\mathrm{h}}^{\text {i.s. }}$ (Sect. 3.2 and Fig. 5 here; Skopal et al. 2006; Burmeister \& Leedjärv 2007) can also be a result of the disk-like structure of the hot object. The scattering process acts on the neutral atoms of hydrogen in the wind from the giant. So, if the ionizing source is indeed capable of producing the $\mathrm{O}^{+5}$ ions (note that the ionization potential $\chi\left(\mathrm{O}^{+5}\right) \sim 114 \mathrm{eV}$ requires $T_{\mathrm{h}}^{\text {i.s. }} \sim 114000 \mathrm{~K}$ (Mürset \& Nussbaumer 1994)), but no Raman line is observed, implies that the $\mathrm{O}^{+5}$ zone is probably too small (cf. Appendix $\mathrm{B}$

in Skopal et al. 2006), so that the disk blocks its radiation in directions to the densest parts of the neutral wind at the orbital plane. However, the arise of a faint redward-shifted Raman line on our spectra from 27/12/2006 and 30/01/2007 (Fig. 5) can be interpreted as due to the Raman scattering in the outer parts of the neutral giant's wind that move from the original O VI photons. This signals a gradual dilution of the disk. Following development of a strong Raman emission with a typical blue-shifted shoulder in the profile (see Fig. 5) reflects total dilution of the optically thick material at the orbital plane, because the original O VI photons then can be scattered in the densest part of the neutral wind from the giant along the binary axis that moves against them (see also Schmid et al. 1999, for a general interpretation of the Raman line profile in symbiotic stars). The simultaneous disappearance of the disk and jets (cf. Figs. 4 and 5) is consistent with the necessity of the disk for the presence of jets (e.g. Livio 1997). The disk-jet connection is discussed below in Sect. 4.

Finally, the disk-like structure of the hot active object in Z And was indicated for the first time by the model SEDs from the 1985 outburst, which revealed directly the presence of the twotemperature UV spectrum (Sect. 5.3.4. of Skopal 2005).

\subsection{Measured parameters of the satellite components}

To isolate the jet components from the whole line profile, we formally fitted the emission line core and its extended foot component with two Gaussian functions. Then the residual jet emissions were compared with additional Gaussians. From their fitted parameters (the central wavelength, maximum, $I$, and the width $\sigma$ ) we derived the jet's radial velocity $R V_{\mathrm{S}}$, flux $F_{\mathrm{S}}=\sqrt{2 \pi} I \sigma$ and the width $F W H M_{\mathrm{S}}=2 \sqrt{2 \ln (2)} \sigma$ (Table 2). Corresponding fits are shown in Fig. 4. This approach and the resolution of our spectra allowed us to estimate uncertainties in the $R V_{\mathrm{S}}$ values to $10-23 \mathrm{~km} \mathrm{~s}^{-1}$ in $\mathrm{H} \alpha, F W H M_{\mathrm{S}}$ widths were possible to adjust to observations within $0.2-0.3 \AA$ and fluxes within 10-20\% of the observed values. The smaller uncertainty corresponds to the stronger jet feature and vice versa. 

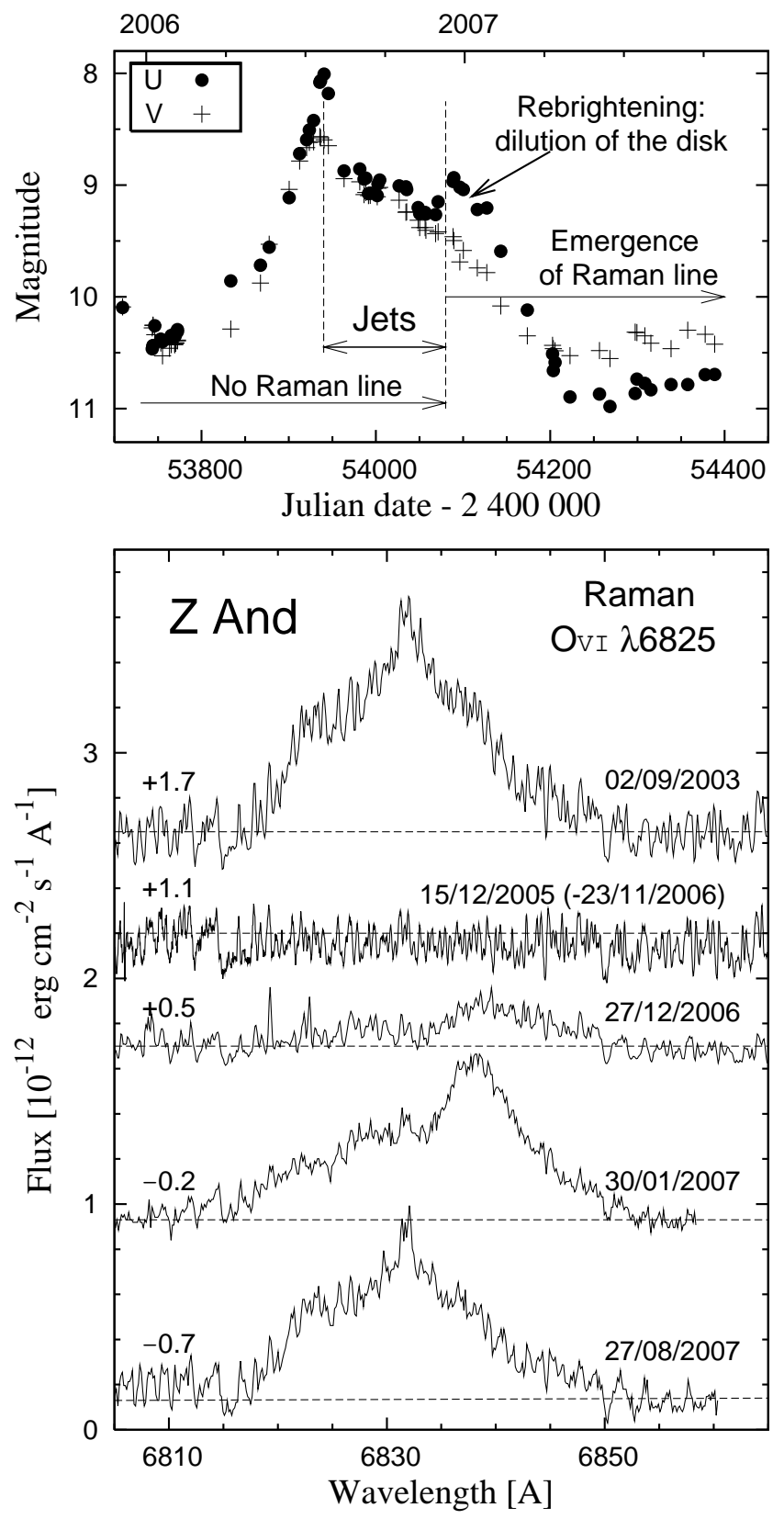

Fig. 5.- Evolution of the Raman-scattered O VI 1032 line throughout the 2006 outburst. Top panel displays the $U$ and $V$ LCs with important periods in evolution of the Raman line, disk and jets. Bottom panel shows variation in the Raman line-profile along the outburst. Disappearance of the Raman line at high ionization temperature during active phases signals the presence of a large optically thick disk around the accretor (15/12/2005 - 23/11/2006). Its emergence (from 27/12/2006) indicates the dilution of the disk (see Sect. 3.3 in detail). Numbers at the left side represent shifts with respect to the level of the local continuum. 
Our observations and those published by Burmeister \& Leedjärv (2007) and Tomov et al. (2007) show an interesting behavior in $R V_{\mathrm{S}}$. From the jets launching in 2006 July to the beginning of 2006 August, the satellite components were located asymmetrically, being shifted by about $+150 \mathrm{~km} \mathrm{~s}^{-1}$ from the symmetric position (the top panel of Fig. 6). Their average velocity was $1280 \mathrm{~km} \mathrm{~s}^{-1}$. Then, in the time of a few days, the jets became symmetric in their velocities. They had persisted at $\left|R V_{\mathrm{S}}\right| \sim 1200 \mathrm{~km} \mathrm{~s}^{-1}$ to about mid of September when reduced suddenly their $\left|R V_{\mathrm{S}}\right|$ to $\sim 1100 \mathrm{~km} \mathrm{~s}^{-1}$ till the end of their detection. The middle panel of Fig. 6 plots the velocity ratio of the faster jet to its slower counterpart that reflects directly the evolution in the jets position. At the initial stage of the jets presence, this ratio was 1.2-1.3, whereas during the following period the velocity ratio was $\approx 1$. The bottom panel of Fig. 6 shows the flux ratio of the red jet component to the blue one, $F_{S^{+}} / F_{S^{-}}$, for the $\mathrm{H} \alpha$ line as a function of the orbital phase. We interpret the decrease of this ratio, indicated around the inferior conjunction of the giant, as an occultation effect (Sect. 3.8.1).

Relatively small width of the satellite components $\left(F W H M_{\mathrm{S}} \sim 250 \mathrm{~km} \mathrm{~s}^{-1}\right.$, Table 2) with respect to, for example, the broad wings of the $\mathrm{H} \alpha$ line $\left(F W Z I(\mathrm{H} \alpha) \sim 4000 \mathrm{~km} \mathrm{~s}^{-1}\right.$, e.g. Fig. 4) implies that (i) the jets are highly collimated, and (ii) the emitting particles can be characterized with one constant velocity within the visible part of the jet. In the following two subsections we discuss some consequences of these jet properties.

\subsubsection{Collimated wind or jets?}

The winds of luminous hot stars are accelerated by the radiation field, produced by the underlying photosphere (e.g. Lamers, \& Cassinelli 1999). They are called line driven winds, because they are driven by absorption in spectral lines. In this process the photons transfer their energy into the kinetic energy of the wind, and, due to the Doppler shifts in the radial direction, the process acts throughout the entire wind, accelerating gradually its particles from the zero to the terminal velocity, $v_{\infty}$. As a result, we observe broad emission lines in the spectra of these stars. For symbiotic binaries, Skopal (2006) suggested that the broad wings of the H $\alpha$ profiles $\left(v_{\infty} \gtrsim 2000 \mathrm{~km} \mathrm{~s}^{-1}\right)$ are formed in the stellar wind from their hot components.

The relatively narrow width of the jet features suggests that they are driven by a different mechanisms. If the jets were driven like the wind, their observed profile would be a few thousands $\mathrm{km} \mathrm{s}^{-1}$ broad, because of the large velocity dispersion in the radial direction $\left(0-v_{\infty}\right)$, and thus would hardly be detectable due to their low emission. Qualitatively, the narrow jet profiles imply that the jet engine works like a gun accelerating material through a short path (relatively to the jet length) to a high velocity, at which the shot-out-material continues to further distances. Also the connection between the disk and the jets, we discuss in Sect. 4, suggests that the jet launching mechanism is very different from that accelerating the wind. Generally, the jet engine transforms the kinetic energy of the inner parts of the disk to the kinetic energy of the flow in the vertical

direction. In this respect, Soker \& Regev (2003) and Soker \& Lasota (2004) suggested a scenario 
how jets could be blown from accretion disks.

\subsubsection{Geometry of the jet emitting region}

The narrow $F W H M_{\mathrm{S}}$ allow us to assume a constant velocity of the emitting particles within the visible part of the jet. Then, if the broadening of the satellite components is caused by thermal motions, the corresponding Doppler width $\left(\sim 250 \mathrm{~km} \mathrm{~s}^{-1}\right.$, i.e. $\sim 5.5 \AA$ in $\left.\mathrm{H} \alpha\right)$ would require unrealistically high temperature of a few millions of Kelvins. In addition, the profiles of satellite lines have sharp edges, do not have typical wings, and their peaks are often flat (Fig. 1, e.g. 10/09/2006). If the jet consisted of a parallel beam of particles, i.e. having the geometry of a narrow column, we could not observe any real broadening. Therefore, we ascribe the observed line width to the dispersion in the line-of-sight velocity components of the jet particles. This can be satisfied if the jet emitting medium has the geometry of a narrow conus with the peak at the central object and characterized with a small opening angle $\theta_{0}$.

Table 2: Parameters of Gaussian fits to the jet emission components: Radial velocity, $R V_{\mathrm{S}}\left[\mathrm{km} \mathrm{s}^{-1}\right]$, flux, $F_{\mathrm{S}}$ $\left[10^{-12} \mathrm{erg} \mathrm{cm}^{-2} \mathrm{~s}^{-1}\right]$ and $F W H M_{\mathrm{S}}\left[\mathrm{km} \mathrm{s}^{-1}\right]$. The level of the local continuum, $F_{\text {cont }}\left[10^{-12} \mathrm{erg} \mathrm{cm}^{-2} \mathrm{~s}^{-1} \AA^{-1}\right]$, opening angle $\theta_{0}\left[^{\circ}\right]$, emission measure of both jets, $E M_{\text {jet }}\left[10^{58} \mathrm{~cm}^{-3}\right]$ (Eq. 5), the mass loss rate through jets scaled to $R_{\text {jet }}=1 \mathrm{AU}, \dot{M}_{\text {jet }}$ (Eq. (9)) and the wind, $\dot{M}_{\mathrm{W}}$ (Sect. 3.9) in $10^{-6} M_{\odot} \mathrm{yr}^{-1}$, are also included.

\begin{tabular}{|c|c|c|c|c|c|c|c|c|c|c|c|c|c|c|}
\hline \multirow{2}{*}{$\begin{array}{c}\text { Date } \\
\mathrm{dd} / \mathrm{mm} / \mathrm{yy}\end{array}$} & \multirow[t]{2}{*}{ Line } & \multicolumn{2}{|c|}{$\overline{R R V_{\mathrm{S}}}$} & \multicolumn{2}{|r|}{$\overline{F F_{\mathrm{S}}}$} & \multicolumn{2}{|c|}{$\overline{F W H} \overline{C M}_{\mathrm{S}}$} & \multirow[t]{2}{*}{$\overline{F F_{\text {cont }}}$} & \multicolumn{2}{|c|}{$\overline{\overline{\theta_{0}}}$} & \multirow[t]{2}{*}{$\overline{E M_{\text {jet }}}$} & \multicolumn{2}{|c|}{$\overline{M_{\text {jet }}}$} & \multirow[t]{2}{*}{$\overline{M_{\mathrm{W}}}$} \\
\hline & & $S^{-}$ & $S^{+}$ & $S^{-}$ & $S^{+}$ & $S^{-}$ & $S^{+}$ & & $S^{-}$ & $S^{+}$ & & $S^{-}$ & $S^{+}$ & \\
\hline $15 / 12 / 05$ & $\mathrm{H} \alpha$ & & no & $\overline{\text { jets } \mathrm{d}}$ & detected & & & 1.0 & - & - & - & - & - & 1.3 \\
\hline $03 / 06 / 06$ & $\mathrm{H} \alpha$ & & no & jets $\mathrm{d}$ & detected & & & 1.6 & - & - & - & - & - & 0.57 \\
\hline $25 / 07 / 06$ & $\mathrm{H} \alpha$ & -1120 & +1385 & - & 8.2 & - & 355 & 2.3 & - & 7.3 & 1.2 & - & 1.7 & - \\
\hline $01 / 08 / 06$ & $\mathrm{H} \beta$ & -1100 & +1408 & 1.1 & 1.9 & 253 & 289 & 3.6 & 6.6 & 5.9 & 1.2 & 0.76 & 1.1 & - \\
\hline \multirow[t]{2}{*}{$30 / 08 / 06$} & $\mathrm{H} \alpha$ & -1250 & +1214 & 5.3 & 5.5 & 227 & 255 & 1.9 & 5.2 & 6.0 & 1.6 & 0.91 & 1.0 & 2.5 \\
\hline & $\mathrm{H} \beta$ & -1267 & +1212 & 2.2 & 2.3 & 204 & 225 & 2.8 & 4.6 & 5.3 & 1.8 & 0.86 & 0.97 & - \\
\hline \multirow[t]{2}{*}{$10 / 09 / 06$} & $\mathrm{H} \alpha$ & -1152 & +1208 & 5.1 & 4.8 & 301 & 280 & 1.7 & 7.5 & 6.6 & 1.5 & 1.2 & 1.1 & 1.5 \\
\hline & $\mathrm{H} \beta$ & -1164 & +1216 & 3.1 & 2.7 & 275 & 275 & 2.6 & 6.7 & 6.5 & 2.3 & 1.4 & 1.3 & - \\
\hline $18 / 10 / 06$ & $\mathrm{H} \alpha$ & -1156 & +1165 & 4.0 & 2.9 & 237 & 226 & 1.6 & 5.9 & 5.5 & 1.0 & 0.83 & 0.66 & 2.7 \\
\hline $09 / 11 / 06$ & $\mathrm{H} \alpha$ & -1100 & +1100 & 2.1 & 1.7 & 237 & 215 & 1.5 & 6.1 & 5.6 & 0.56 & 0.59 & 0.49 & 2.4 \\
\hline $23 / 11 / 06$ & $\mathrm{H} \alpha$ & -1120 & +1100 & 2.0 & 1.9 & 237 & 215 & 1.3 & 6.0 & 5.6 & 0.57 & 0.58 & 0.52 & 2.7 \\
\hline $27 / 12 / 06$ & $\mathrm{H} \alpha$ & -1110 & +1110 & 0.87 & 0.60 & 247 & 237 & 1.2 & 6.3 & 6.1 & 0.22 & 0.38 & 0.33 & 2.5 \\
\hline $30 / 01 / 07$ & $\mathrm{H} \alpha$ & & no & jets $\mathrm{d}$ & detected & & & 1.1 & - & - & - & - & - & 1.0 \\
\hline $11 / 04 / 07$ & $\mathrm{H} \alpha$ & & no & jets $d$ & detected & & & 0.83 & - & - & - & - & - & 2.5 \\
\hline $22 / 06 / 07$ & $\mathrm{H} \alpha$ & & no & jets $d$ & detected & & & 0.80 & - & - & - & - & - & 1.5 \\
\hline $31 / 07 / 07$ & $\mathrm{H} \alpha$ & & no & jets $d$ & detected & & & 0.84 & - & - & - & - & - & 1.3 \\
\hline $27 / 08 / 07$ & $\mathrm{H} \alpha$ & & no & jets $d$ & detected & & & 0.82 & - & - & - & - & - & 0.78 \\
\hline
\end{tabular}

\subsection{Opening angle of the jets}

The conus geometry of the jet and the constant velocity of its particles (Sect. 3.4.2.) allow us to express the observed width of the satellite component as a function of its opening angle $\theta_{0}$ and the 

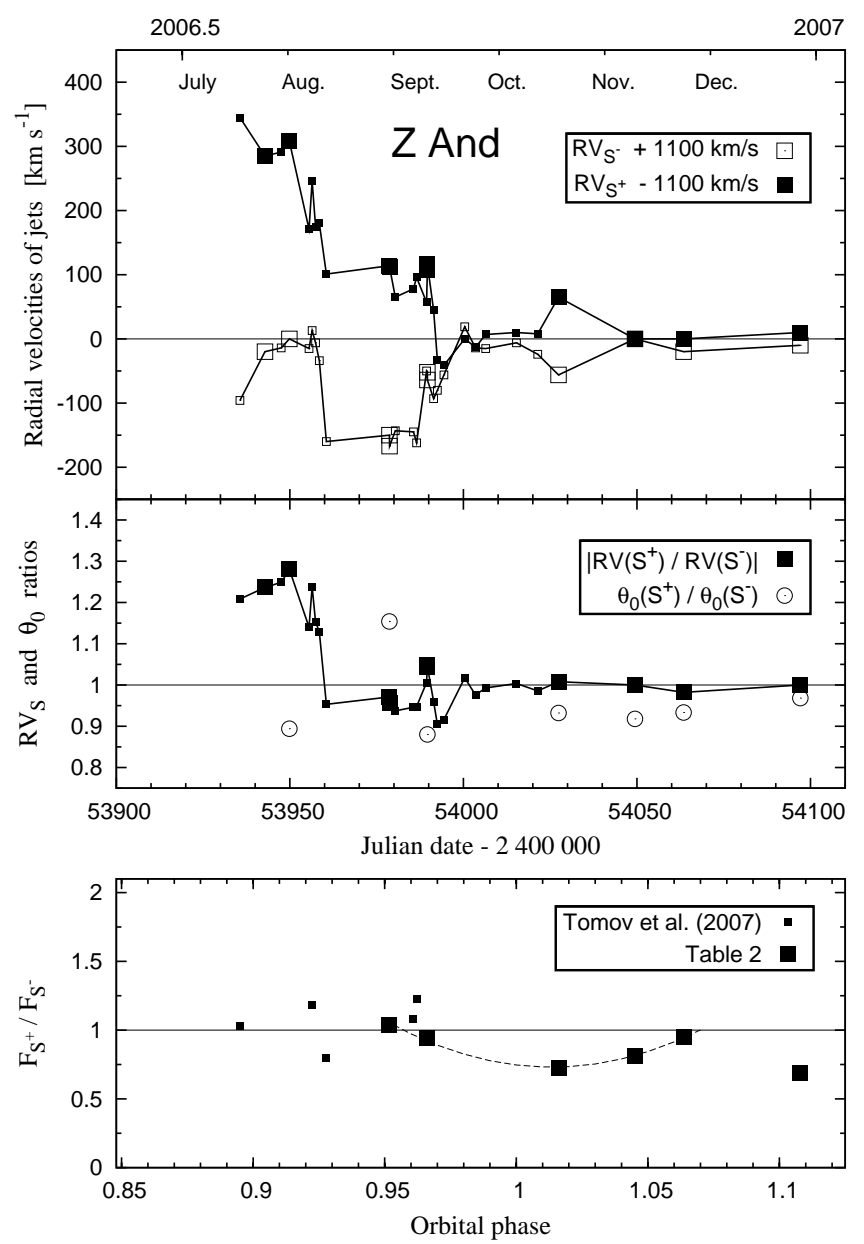

Fig. 6.- Top: evolution of the jet radial velocities, $R V_{\mathrm{S}}$. Large symbols represent our data (Table 2), while small ones are from Burmeister \& Leedjärv (2007) and Tomov et al. (2007) for the H $\alpha$ line. Measured values were shifted by $\pm 1100 \mathrm{~km} \mathrm{~s}^{-1}$ to visualize better their relative position. Middle: $R V_{\mathrm{S}^{+}} / R V_{\mathrm{S}^{-}}$ratios demonstrate the asymmetry in the jet radial velocities at the beginning of their launching. Compared is the ratio of opening angles (Sects. 3.5. and 4.2). Bottom: the ratio of the jet component fluxes, $F_{S^{+}} / F_{S^{-}}$, for the $\mathrm{H} \alpha$ line as a function of the orbital phase. Their transient decrease around the phase 1.0 could be the effect of occultation of the red jet component by the giant (Sect. 3.8.1). 
orbital inclination $i$. Assuming that the jets were launched with the velocity, $v_{\text {jet }}$, perpendicularly to the disk plane that coincides with the orbital one, the maximum dispersion of the line-ofsight jet velocity component corresponds to $2 \times H W Z I_{\mathrm{S}}=v_{\text {jet }} \cos \left(i-\theta_{0} / 2\right)-v_{\text {jet }} \cos \left(i+\theta_{0} / 2\right)=$ $2 \sin (i) \sin \left(\theta_{0} / 2\right)$, where the half width at the zero intensity of the jet $\left(H W Z I_{\mathrm{S}}\right)$ is in $\mathrm{km} \mathrm{s}^{-1}$. Then for the measured central jet's velocity, $R V_{\mathrm{S}}=v_{\text {jet }} \cos (i)$, the opening angle can be approximated as

$$
\theta_{0}=2 \sin ^{-1}\left[\frac{H W Z I_{\mathrm{S}}}{R V_{\mathrm{S}} \tan (i)}\right]
$$

A similar relation was also used by Shahbaz et al. (1997). Corresponding parameters from Table 2, orbital inclination $i=76^{\circ}$ (Skopal 2003) and adopting $H W Z I_{\mathrm{S}}=F W H M_{\mathrm{S}}$, yield its average value as

$$
\theta_{0}=6 .^{\circ} 1 \pm 0.45 \times \Delta i,
$$

where the uncertainty represents a total differential of function (1) for uncertainties in $F W H M_{\mathrm{S}}$, $R V_{\mathrm{S}}$ (Sect. 3.4) and that in the orbital inclination, $\Delta i$.

\subsection{The jet speed and the mass of the accretor}

It is generally considered that the jet velocity in all the jet-producing objects is of the order of the escape velocity from their central stars, which indicates that the jet-type outflows originate from the vicinity of the accretors (e.g. Livio 1997, and references therein). Recently, Soker \& Regev (2003) and Soker \& Lasota (2004) performed analytical estimates on how the plasma close to the central object, specifically in the boundary layer of the disk, could be accelerated to velocities larger than the local escape velocity. Our observations seem to be consistent with this scenario. The high velocities of jets, $v_{\text {jet }} \sim 5000 \mathrm{~km} \mathrm{~s}^{-1}$, observed at large distances from the central star (Sec. 3.8.1), imply $v_{\text {jet }} / v_{\text {escape }}>1$ for all possible distances of the jet ejection. However, from observations it is difficult to determine accurately the distance from the accreting body, at which jets are launched. Soker \& Lasota (2004) also could not rule out the possibility that jets can be launched somewhat away from the accretor, up to $\sim 3 \times$ the boundary layer radius. They noted that this case would require a strong perturbation in the disk.

Therefore, for a rough estimate only, we will assume that the jets were launched from the WD surface at $v_{\text {jet }} \sim v_{\text {escape }}$. Then the average value of $v_{\text {jet }}=R V_{\mathrm{S}} / \cos (i)=4960 \pm 90 \mathrm{~km} \mathrm{~s}^{-1}$ $\left(R V_{\mathrm{S}}=1200 \pm 22 \mathrm{~km} \mathrm{~s}^{-1}\right.$, Table $\left.2, i=76^{\circ}\right)$ and a typical WD radius of $R_{\mathrm{WD}}=0.01 R_{\odot}$ correspond to the mass of the accretor in $\mathrm{Z}$ And, $M_{\mathrm{WD}} \sim 0.64 M_{\odot}$. We note that this value satisfies perfectly the relation between mass and radius of WDs (e.g. Shapiro \& Teukolsky 1983). Schmid \& Schild (1997) derived $M_{\mathrm{WD}}=0.65 \pm 0.28 M_{\odot}$ from the spectroscopic orbit for $i=47^{\circ}$. Our value of $M_{\mathrm{WD}}$ derived for $i=76^{\circ}$ requires just a larger total system mass of $\sim 3.2 M_{\odot}$ to satisfy the mass function of $0.024 M_{\odot}$ (Fekel et al. 2000). However, in the real case, the same value of $M_{\mathrm{WD}}$ can be obtained for different combinations of the $v_{\text {jet }} / v_{\text {escape }}(>1)$ ratio and the distance of the jet ejection. 
Finally, we note that the large effective radius of the active star during the optical maximum derived from the SED $\left(R_{\mathrm{h}}^{\text {eff }} \sim 12 R_{\odot} \gg R_{\mathrm{WD}}\right.$, Sect. 3.1$)$ does not contradict to launching the jets from the vicinity of the WD surface, because of the disk-like structure of the hot active object seen under a high inclination angle (Sect. 3.3).

\subsection{Radiation of jets}

In this section we derive some constraints supporting that the jet emission is due to the photoionization of hydrogen and that the medium is optically thin in the direction of the observer.

(i) According to Fig. 3 of Burmeister \& Leedjärv (2007) and the $F_{\mathrm{S}}$ fluxes from Table 2, the average Balmer decrement $F_{\mathrm{S}}(\mathrm{H} \alpha) / F_{\mathrm{S}}(\mathrm{H} \beta) \sim 2.4$, which is close to the theoretical value of 2.75, given by the recombination process at the electron temperature of $20000 \mathrm{~K}$ (e.g. Gurzadyan 1997). Equivalent widths presented by Burmeister \& Leedjärv were converted to fluxes with the aid of our continuum calibration in Table 2. The somewhat lower values of the observed decrement than the theoretical one could be caused by a partial opacity of the nebular jet medium in the Balmer lines. Generally, the opacity in the $\mathrm{H} \alpha$ line is larger than in the $\mathrm{H} \beta$. The effect, however, should not be significant. Also the high orbital inclination and the small value of $\theta_{0}$ correspond to a relatively small intersection, $l$, of the jet conus with the line of sight, and thus to a low value of the optical depth $(\tau \propto$ opacity $\times l)$, which supports rather optically thin regime in the direction of the observer. For a comparison, the jets produced by the symbiotic star MWC 560 are optically thick, because they are seen nearly pole-on, i.e. their $l$ and thus $\tau$ are very large, which gives rise the blueshifted jet absorption components in the spectrum (Schmid et al. 2001).

(ii) If the jet emission is due to the recombination of free electrons with protons, then it can be created only within the ionized fraction of the ejected material. In Appendix A we calculate the extension of the jet nebula as the distance from the central hot star, at which ionizing photons

are completely consumed by jet particles along paths outward from the ionizing star. According to Eq. (A2) the jet radius is limited by its Strömgren sphere at the distance $r_{\mathrm{S}}$ from the ionizing source, i.e.

$$
R_{\text {jet }} \equiv r_{\mathrm{S}}=\left(\frac{3 L_{\mathrm{ph}}}{4 \pi \alpha_{\mathrm{B}}\left(H, T_{\mathrm{e}}\right)} \bar{n}_{\text {jet }}^{-2}\right)^{1 / 3}
$$

where the parameters, $L_{\mathrm{ph}}, \alpha_{\mathrm{B}}\left(H, T_{\mathrm{e}}\right), \bar{n}_{\text {jet }}$ are explained in Appendix A. In Section 3.8 and Figure 7) we demonstrate that the jets radius, determined independently from their observed luminosity and the opening angle (Eq. (7)), agrees well with the radius of the Strömgren sphere, given by the luminosity and the temperature of the ionizing source. This result confirms that the satellite components are really created by recombinations within the jets, ionized by the central star.

Evolution in the fluxes of jets is consistent with such an ionization structure. Between the beginning of 2006 August and the end of September, their fluxes settled (with some fluctuations) around a maximum (Burmeister \& Leedjärv 2007; Tomov et al. 2007, this paper). No increasing 
trend, as could be expected from an increase of the emission measure of jets (see Eq. (5)) due to their expansion, was observed during this period. This implies that the emitting mass in jets was approximately constant, and thus suggests that the satellite components represent only the illuminated part of jets ionized by the hot central object, which is bounded by the Strömgren sphere. Figure 9 (see Appendix B) shows that the number of hydrogen ionizing photons is approximately constant for temperatures between about 50000 and $130000 \mathrm{~K}$ at a fixed luminosity of a black-body source. Therefore, the hot star produced roughly constant flux of ionizing photons, and thus also relevant fluxes of jets, in spite that its temperature increased from $75000 \mathrm{~K}$ to $115000 \mathrm{~K}$ during the above mentioned period (Burmeister \& Leedjärv 2007). Observed fluctuations were probably caused by those in the luminosity of the ionizing object.

\subsection{Mass loss through the jets}

The recombination process of the radiation by the jet plasma, the resulting fluxes, and the geometrical and kinematics parameters of the jets put some constraints to determine the mass loss rate through jets. Assuming that the jets were expelled into the solid angle $\Delta \Omega=2 \pi\left[1-\cos \left(\theta_{0} / 2\right)\right]$ of the jet nozzle (Sect. 3.4.2.), then the corresponding mass-loss rate, $\dot{M}_{\text {jet }}$, and the mean particle concentration, $\bar{n}_{\text {jet }}$, in the jets volume, are connected via the mass continuity equation as

$$
\dot{M}_{\mathrm{jet}}=\Delta \Omega R_{\mathrm{jet}}^{2} \mu m_{\mathrm{H}} \bar{n}_{\mathrm{jet}} v_{\mathrm{jet}},
$$

where $\mu$ is the mean molecular weight and $m_{\mathrm{H}}$ is the mass of the hydrogen atom. Total luminosity produced by jets through the recombination transition of the $\mathrm{H} \alpha$ line, $L_{\text {jet }}(\mathrm{H} \alpha)$, is related to the line emissivity, $\varepsilon_{\alpha} n_{\mathrm{e}} n_{\mathrm{p}}\left(\mathrm{erg} \mathrm{cm}^{-3} \mathrm{~s}^{-1}\right)$, by

$$
L_{\text {jet }}(\mathrm{H} \alpha)=\varepsilon_{\alpha} \int_{V_{\text {jet }}} n_{\mathrm{e}} n_{\mathrm{p}} \mathrm{d} V \equiv \varepsilon_{\alpha} \bar{n}_{\text {jet }}^{2} V_{\text {jet }}=\varepsilon_{\alpha} E M_{\text {jet }},
$$

where $\varepsilon_{\alpha}$ is the volume emission coefficient in $\mathrm{H} \alpha, n_{\mathrm{e}}$ and $n_{\mathrm{p}}$ are concentrations of electrons and protons and $V_{\text {jet }}$ and $E M_{\text {jet }}$ are the volume and emission measure of the jets, respectively. For the optically thin medium of jets, the luminosity can be determined from the observed fluxes as $L_{\text {jet }}=4 \pi d^{2} \times F_{\mathrm{S}}$. Further, Eq. (5) assumes a completely ionized medium (i.e. $n_{\mathrm{e}}=n_{\mathrm{p}} \equiv \bar{n}_{\text {jet }}$ ), radiating at a constant electron temperature, i.e. $\varepsilon_{\alpha}$ is constant throughout the jet emitting volume. According to Sect. 3.4.2. we approximate its geometry by the conus, i.e.

$$
V_{\text {jet }}=\frac{1}{3} R_{\text {jet }}^{3} \times \Delta \Omega
$$

Substituting Eq. (6) into Eq. (5), we can express the jet radius by means of the parameters, obtained directly from observations $\left(L_{\text {jet }}\right.$ and $\Delta \Omega$ ), as

$$
R_{\text {jet }}=\left(\frac{3 L_{\text {jet }}(\mathrm{H} \alpha)}{\varepsilon_{\alpha}\left(H, T_{\mathrm{e}}\right) \Delta \Omega} \bar{n}_{\text {jet }}^{-2}\right)^{1 / 3} .
$$


This expression allows us to rewrite Eq. (4) as

$$
\dot{M}_{\text {jet }}=\xi_{1} \times\left(\frac{\Delta \Omega}{\bar{n}_{\text {jet }}}\right)^{1 / 3}\left(\frac{L_{\text {jet }}}{\left[L_{\odot}\right]}\right)^{2 / 3} \frac{v_{\text {jet }}}{\left[\mathrm{km} \mathrm{s}^{-1}\right]} M_{\odot \mathrm{yr}^{-1}}
$$

and/or as a function of the radius $R_{\text {jet }}$ as

$$
\dot{M}_{\text {jet }}=\xi_{2} \times\left(\frac{R_{\text {jet }}}{[A U]} \frac{\Delta \Omega}{[\mathrm{sr}]} \frac{L_{\text {jet }}}{\left[L_{\odot}\right]}\right)^{1 / 2} \frac{v_{\text {jet }}}{\left[\mathrm{km} \mathrm{s}^{-1}\right]} M_{\odot} \mathrm{yr}^{-1},
$$

where the factor $\xi_{1}=5.9 \times 10^{-6}$ or $1.1 \times 10^{-5}$ and $\xi_{2}=3.6 \times 10^{-9}$ or $5.9 \times 10^{-9}$ for luminosities in $\mathrm{H} \alpha$ or $\mathrm{H} \beta$, respectively. Volume emission coefficients for $T_{\mathrm{e}}=2 \times 10^{4} \mathrm{~K}, \varepsilon_{\alpha}=1.83$ and $\varepsilon_{\beta}=$ $0.682 \times 10^{-25} \mathrm{erg} \mathrm{cm}^{3} \mathrm{~s}^{-1}$ (Osterbrock 1989).

\subsubsection{Additional constraints for $\dot{M}_{\text {jet }}$}

According to Eqs. (8) and (9), determination of $\dot{M}_{\text {jet }}$ requires to estimate reasonable values of $\bar{n}_{\text {jet }}$ or $R_{\text {jet }}$. Figure 7 plots dependencies between these parameters. Below we discuss some constraints that allow us to determine more accurate ranges of the jet parameters.

(i) Soker \& Lasota (2004) introduced conditions for thermally launching jets from accretion disks around WDs. In their model the accreted material is strongly shocked due to large gradients of physical quantities in the boundary layer and cools on the timescale longer than its ejection time from the disk. The model requires large accretion rates of $\dot{M}_{\text {acc }} \gtrsim 10^{-6} M_{\odot} \mathrm{yr}^{-1}$. Some observational evidences of the disk-jet connection, as described in Section 4, could support this scenario. In agreement with this suggestion and the fact that there is a large disk encompassing the WD (Sects. 3.1. and 3.3.), we can exclude rates of $\dot{M}_{\text {jet }} \lesssim 5.5 \times 10^{-7} M_{\odot} \mathrm{yr}^{-1}$ (i.e. $\dot{M}_{\text {acc }} \lesssim$ $5.5 \times 10^{-6} M_{\odot} \mathrm{yr}^{-1}$, Sect. 4.1 ) that correspond to $\bar{n}_{\text {jet }} \gtrsim 10^{12} \mathrm{~cm}^{-3}$ and $R_{\text {jet }} \lesssim 0.086 \mathrm{AU}$ (see Fig. 7), because of too small jet radii that would be significantly occulted by the disk for the orbital inclination of $\sim 75^{\circ}$. Observations do not indicate this case (e.g. Table 2, Fig. 6). From the other side, we exclude rates $\dot{M}_{\text {jet }} \gtrsim 5.5 \times 10^{-6} M_{\odot} \mathrm{yr}^{-1}$ (i.e. $\bar{n}_{\text {jet }} \lesssim 10^{9} \mathrm{~cm}^{-3}, R_{\text {jet }} \gtrsim 9$ AU, Fig. 7 ), because this would require too high accretion rates of $\gtrsim 5.5 \times 10^{-5} M_{\odot} \mathrm{yr}^{-1}$ (see also Sect. 4.1). As the mass of the disk can be estimated to $\sim 10^{-4}-10^{-5} M_{\odot}$ (e.g. $R_{\mathrm{D}}=20 R_{\odot}, H / R_{\mathrm{D}}=0.3$ (Skopal 2006) and average density $\log \rho=-7$ to -8 ), the accretion rates of about $10^{-4} M_{\odot} \mathrm{yr}^{-1}$ would exhaust the disk during a short time. However, observations indicate the presence of a large disk with jets for about six months, from 2006 July to 2007 January (Sect. 3.3, Fig. 5). These conditions thus limit $\dot{M}_{\text {jet }}$ to $5.5 \times 10^{-6}>\dot{M}_{\text {jet }}>5.5 \times 10^{-7} M_{\odot} \mathrm{yr}^{-1}$ and consequently, $10^{9}<\bar{n}_{\text {jet }}<10^{12} \mathrm{~cm}^{-3}$ and $0.1<R_{\text {jet }}<9 \mathrm{AU}$.

(ii) During active phases of $\mathrm{Z}$ And the emission measure produced by the ionized hot star wind was determined to $E M_{\mathrm{W}} \sim 3 \times 10^{59} \mathrm{~cm}^{-3}$ (Table 1 in Skopal 2006). It represents the socalled low-temperature nebula, which is subject to eclipses in active symbiotic systems (Skopal 
2005, 2006), and thus it is located within the radius of the giant, $R_{\mathrm{G}}$, around the hot component. For $\mathrm{Z}$ And, the average particle concentration in the wind around the hot star within the radius $R_{\mathrm{G}}=106 R_{\odot}$ is $\bar{n}_{\mathrm{W}}=\left(E M_{\mathrm{W}} / V_{\mathrm{G}}\right)^{1 / 2}=1.3 \times 10^{10} \mathrm{~cm}^{-3}$. For a comparison, the mean concentration of the jet particles within this distance, $\bar{n}_{\text {jet }}(0.5 \mathrm{AU}) \sim 7.5 \times 10^{10} \mathrm{~cm}^{-3}$, which corresponds to $\dot{M}_{\text {jet }} \sim 1.3 \times 10^{-6} M_{\odot} \mathrm{yr}^{-1}$ (Fig. 7). This suggests that $\bar{n}_{\text {jet }}>\bar{n}_{\mathrm{W}}$ and also the normalized mass loss rate (e.g. into $1 \mathrm{sr}$ ) via the jets is larger than that through the wind.

(iii) The transient decrease in the flux ratio, $F_{\mathrm{S}^{+}} / F_{\mathrm{S}^{-}}<1$, measured during the inferior conjunction of the giant (Fig. 6), could be caused by the occultation of the red jet component by the stellar disk of the giant (also noted by Skopal \& Wolf 2006). As $2 R_{\mathrm{G}} \sim 1 \mathrm{AU}$, this observation suggests that the radii of jets are $\gtrsim 1$ AU. However, uncertainties in the orbital elements do not allow a more accurate estimate (see Formiggini \& Leibowitz 1994; Mikolajewska \& Kenyon 1996; Skopal 1998; Fekel et al. 2000). Finally, we note that some theoretical works, devoted to modeling the jets in symbiotic stars, also adopt the jet radius of $1 \mathrm{AU}$ (e.g. Stute \& Camenzind 2005; Stute \& Sahai 2007). Therefore, we determined the mass loss rate $\dot{M}_{\text {jet }}$ in Table 2 for a representative value of $R_{\text {jet }} \equiv 1 \mathrm{AU}$.

\subsubsection{Emitting mass in jets}

The mean concentration and the volume of jets determine their emitting mass as

$$
M_{\mathrm{jet}}^{e m}=\mu m_{\mathrm{H}} \bar{n}_{\mathrm{jet}} V_{\mathrm{jet}},
$$

which can be rewritten with the aid of Eqs. (6) and (7) as

$$
M_{\text {jet }}^{e m}=\xi_{3} \times\left(\frac{\Delta \Omega}{[\mathrm{sr}]} \frac{L_{\text {jet }}}{\left[L_{\odot}\right]}\right)^{1 / 2}\left(\frac{R_{\text {jet }}}{[A U]}\right)^{3 / 2} M_{\odot},
$$

where $\xi_{3}=5.7 \times 10^{-9}$ or $9.3 \times 10^{-9}$ for luminosities in $\mathrm{H} \alpha$ or $\mathrm{H} \beta$, respectively. The average value of $\theta_{0}=6 .^{\circ} 1$ (Sect. 3.5), $L_{\text {jet }}=0.32 L_{\odot}$ in $\mathrm{H} \alpha$ (from the 2006 August - September maximum) and $R_{\text {jet }}$ $=1 \mathrm{AU}$ yield the emitting mass of $M_{\text {jet }}^{e m}=6 \times 10^{-10} M_{\odot}$ in both jet components. Tomov et al. (2007) derived significantly larger value of $M_{\text {jet }}^{e m}=1.4 \times 10^{-7} M_{\odot}$ for the similar luminosity of the jet pair they observed on 12/08/2006. This difference results from their adopted mean density of

only $10^{8} \mathrm{~cm}^{-3}$, which requires very large size of one jet, $R_{\text {jet }} \doteq 40 \mathrm{AU}$. However, such an extension of the jet is beyond the limits we estimated in Sect. 3.8.

\subsubsection{Total mass released by jets}

With respect to our approximation, in which the jet medium has a constant particle density and emits within its Strömgren radius, we approximate the total mass released by the jets during 
the time of their detection, $\Delta T_{\text {jet }}$, as

$$
M_{\text {jet }}^{\text {total }} \approx \widehat{\dot{M}_{\text {jet }}} \times \Delta T_{\text {jet }}
$$

where $\widehat{\dot{M}_{\text {jet }}}$ is the average mass loss rate via jets. Values from Table $2\left(\widehat{\dot{M}_{\text {jet }}}=1.75 \times 10^{-6} M_{\odot} \mathrm{yr}^{-1}\right.$ for the jet pair and $\Delta T_{\text {jet }}=154$ days) yield $M_{\text {jet }}^{\text {total }} \approx 7.4 \times 10^{-7} M_{\odot}$.

\subsection{Mass loss through the wind}

It is generally well known that during active phases the hot components in symbiotic binaries lose their mass in the form of wind. To estimate the rate of the mass loss produced by the active star through the stellar wind, we used the $\mathrm{H} \alpha$ method as suggested by Skopal (2006). The method assumes that the broad wings are due to kinematics of the photoionized and optically thin stellar wind. Profiles from the maximum were modeled with the same input parameters as those from the 2000-maximum. The disk radius, $R_{\mathrm{D}}=6.4 R_{\odot}$, and the disk thickness at its edge, $H=1.92 R_{\odot}$, were adjusted to the effective radius of the hot object derived from the SED assuming a flared disk with $H / R_{\mathrm{D}}=0.3$. Prior to and after the outburst we used $R_{\mathrm{D}}=3.5 R_{\odot}$ and $H=1.05 R_{\odot}$. The results do not depend critically on these parameters. Examples of comparison between the modeled and observed profiles are shown in Figs. 4 and 8. Synthetic profiles fit well the observed wings for $|R V| \gtrsim 300 \mathrm{~km} \mathrm{~s}^{-1}$. Corresponding mass loss rates through the wind, $\dot{M}_{\mathrm{W}}$ are around $2 \times 10^{-6} M_{\odot} \mathrm{yr}^{-1}$ (Table 2). Other model parameters are the terminal velocity, which runs between $1500 \mathrm{~km} \mathrm{~s}^{-1}$ and $3000 \mathrm{~km} \mathrm{~s}^{-1}$, and the acceleration parameter $\beta \sim 1.7$ (cf. Eq. (2) in Skopal 2006).

It is of interest to note that the observation made just prior to the outbursts (the HET spectrum) revealed a more complex structure in the wind outflow. The $O-C$ plot shows a faint and very broad $\left(\mathrm{FWHM} \approx 1200 \mathrm{~km} \mathrm{~s}^{-1}\right.$ ) emission component superposed on the extended blue $\mathrm{H} \alpha$ wing (Fig. 8).

\section{A disk-jet connection}

\subsection{Observational evidence}

The red giant in $\mathrm{Z}$ And loses mass via the wind at $\dot{M}_{\mathrm{G}} \sim 7 \times 10^{-7} M_{\odot} \mathrm{yr}^{-1}$ (Skopal 2005, Table 3). According to the recent study on the wind accretion in binary stars (Nagae et al. 2004) the WD companion can accrete with efficiency up to $10 \%$, i.e. at $\dot{M}_{\text {acc }}^{\text {wind }} \sim 7 \times 10^{-8} M_{\odot} \mathrm{yr}^{-1}$ during quiescent phases of $\mathrm{Z}$ And (the total system mass of $3.2 M_{\odot}$ and the wind velocity of $30 \mathrm{~km} \mathrm{~s}^{-1}$ at the accretor were adopted). This quantity corresponds to the accretion luminosity of $\sim 70 L_{\odot}$ (parameters from Sect. 3.6), which is at least a factor of 30 smaller than the observed luminosity of the hot stellar object (e.g. Skopal 2005). This discrepancy led to suggestion that an additional 


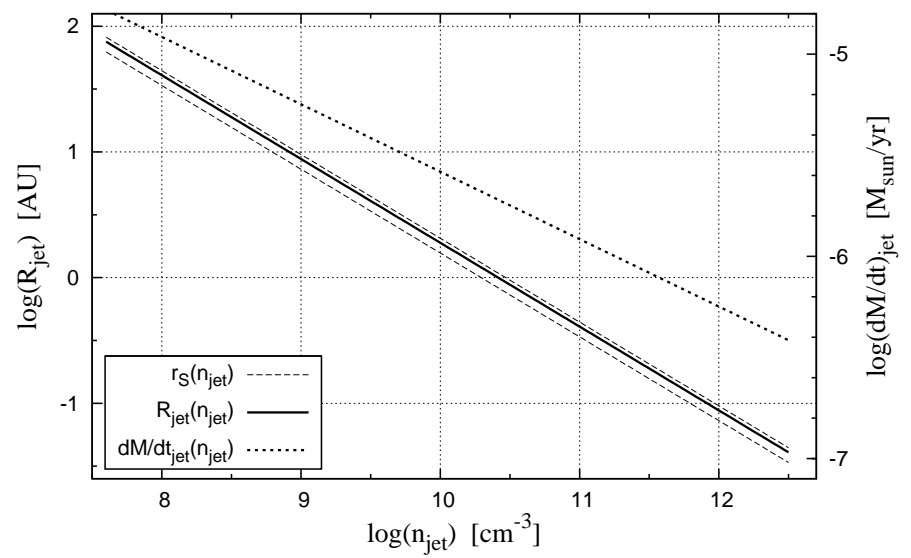

Fig. 7.- Dependencies of $R_{\text {jet }}$ (Eq. (7)) and $\dot{M}_{\text {jet }}$ (Eq. (8)) on $\bar{n}_{\text {jet. }}$ They were calculated for average values of $L_{\text {jet }}=0.32 L_{\odot}$ (to the end of 2006 Sept.) and $\theta_{0}=6 .^{\circ} 1$ (i.e. $\Delta \Omega=8.9 \times 10^{-3}$ sr). $\dot{M}_{\text {jet }}$ here represents the mass loss rate by both the jets. Compared are radii of the Strömgren sphere $r_{\mathrm{S}}$ (Eq. (3)) for the hot star luminosity of $10^{4} L_{\odot}$, estimated by Sokoloski et al. (2006) (lower line), and for $2.2 \times 10^{4} L_{\odot}$ as results for scaling this quantity to our parameters of $d=1.5 \mathrm{kpc}$ and $E_{\mathrm{B}-\mathrm{V}}=0.30$ (upper line). A good agreement between both $R_{\text {jet }}$ and $r_{\mathrm{S}}$ radii confirms the recombination process as responsible for the radiation of the jet plasma, ionized by the central star.

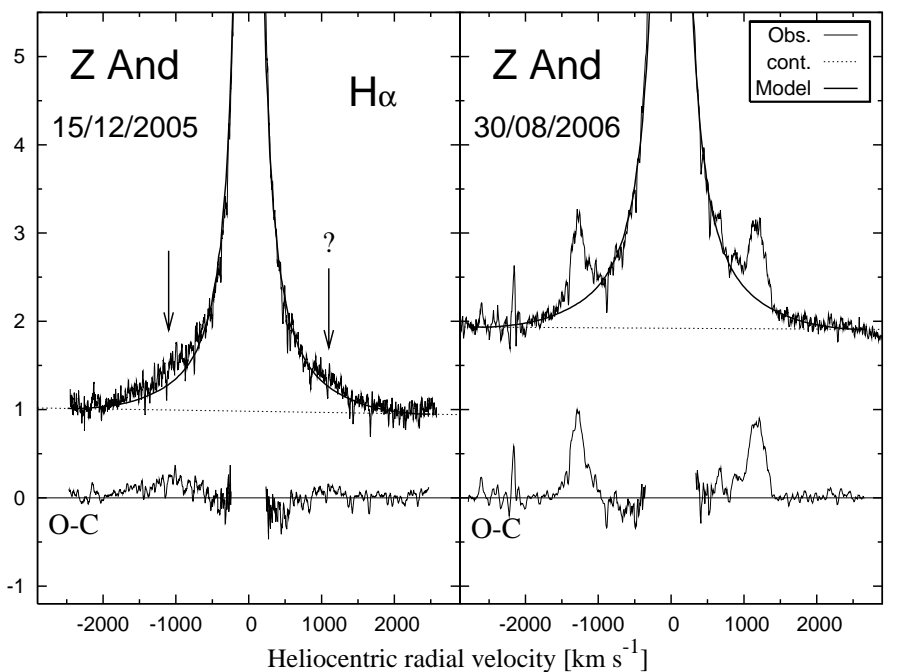

Fig. 8.- Two examples of the observed and synthetic $\mathrm{H} \alpha$ profiles from the ionized stellar wind of the hot star. The $O-C$ plot demonstrates a good fit for $|R V|>300 \mathrm{~km} \mathrm{~s}^{-1}$. The models correspond to the mass loss rate of 1.3 and $2.5 \times 10^{-6} M_{\odot} \mathrm{yr}^{-1}$ for $15 / 12 / 2005$ and $30 / 08 / 2006$, respectively, $v_{\infty}=2700 \mathrm{~km} \mathrm{~s}^{-1}$ and $\beta=1.7$ (Sect. 3.9). Fluxes are in $10^{-12} \mathrm{erg} \mathrm{cm}^{-2} \mathrm{~s}^{-1} \AA^{-1}$. 
source of the energy in a form of steadily burning material on the white dwarf surface has to be present (e.g. Mikolajewska \& Kenyon 1992).

During the recent active phase, the $\dot{M}_{\text {jet }}$ rate put limits for the accretion rate through the disk, $\dot{M}_{\text {acc }}$, because both the rates are proportional. Typical values of $\dot{M}_{\text {jet }} / \dot{M}_{\text {acc }}$ are in the range 0.01-0.3 (e.g. Lizano et al. 1988; Pringle 1993; Livio 1997), although Livio et al. (2003) found that $\dot{M}_{\text {jet }}$ can almost be equal to $\dot{M}_{\text {acc }}$, if the accretion energy is converted efficiently into magnetic energy and is emitted in the form of a magnetically dominated outflow or jet. Here we will consider the ratio $\dot{M}_{\text {jet }} / \dot{M}_{\text {acc }} \sim 0.1$. Accordingly, the observed $\dot{M}_{\text {jet }} \sim 10^{-6}\left(R_{\text {jet }} / 1 \mathrm{AU}\right)^{1 / 2} M_{\odot} \mathrm{yr}^{-1}$ thus requires $\dot{M}_{\text {acc }} \sim 10^{-5} M_{\odot} \mathrm{yr}^{-1}$. Two possible mechanisms how to increase the accretion rate are either a disk instability or an increase in the mass loss rate from the giant. If the latter possibility had been the case, the normal red giant in $\mathrm{Z}$ And would have increased its $\dot{M}_{\mathrm{G}}$ by 3 orders of magnitude - from a few times $10^{-7} M_{\odot} \mathrm{yr}^{-1}$ during quiescence to $\approx 10^{-4} M_{\odot} \mathrm{yr}^{-1}$ for a short time during the activity, to power the observed jets. This seems to be unlikely. Thus, assuming that $\dot{M}_{\mathrm{G}}$ is more or less constant, a disk instability had to be responsible for the transient, but significant, increase in $\dot{M}_{\text {acc }}$ through the disk. We summarize critical observations supporting this suggestion as follows:

1. The rapid photometric variability originates in the disk (Sects. 3.1 and 3.3). Figure 3 shows the change from an irregular low-amplitude variation prior to the outburst to a slower, but higher-magnitude variation, observed during the maximum when the jets were launched. According to Sokoloski \& Kenyon (2003), who examined the disk-jet connection in CH Cyg, the disappearance of the fastest variations indicates that the innermost disk was disrupted around the time when the jets were produced. This interpretation is based on the relationship between the disk radius and variability timescale (slower variations come from larger disk radii and vice versa, as summarized by Sokoloski \& Kenyon 2003). Also the gas-dynamical modeling of the flow structures during the Z And outburst suggested that the sudden increase of the accretion rate could result from the disruption of the disk (Bisikalo et al. 2006). The disk disruption, i.e. the removal of material from its inner parts, implies that the jets were accretion-powered.

2. Dramatic spectroscopic evolution around the optical maximum (2006 July/August), as indicated by complex absorption/emission profiles accompanied by the asymmetric ejection of jets (Fig. 4, Sect. 3.2 here and Fig. 2 in Tomov et al. 2007), could also be a direct result of the disk disruption. It is of interest to note that similar behavior was also observed during CH Cyg outbursts in 1982-84, 1992-94 and 1998-00, but on somewhat longer timescales (see Figs. 1 and 4 of Skopal et al. 2002). All the outbursts were followed by jet-extended features on the radio images (Crocker et al. 2001). However, for Z And these features have been recorded for the first time. For example, during its 2000-03 major outburst no jets were present (see Sokoloski et al. 2006; Skopal et al. 2006).

3. Simultaneous presence of both the disk and jets during the period 2006 July - December and 
their disappearance from 2007 January (Sect. 3.3, Figs. 4 and 5) suggest that bulk of the accretion energy was released in the form of jets. As a result the hot stellar source in Z And had reduced its radiation significantly and therefore we observed a rapid decline in the star's brightness from 2007 January (see Figs. 1 and 5). From this point of view, the absence of jets during the previous (2000-03) major outburst is consistent with a slower decline in the brightness from the 2000-maximum (see Fig. 1) and the disk presence for a longer time $(\sim 1.5$ years, Skopal et al. 2006). In both the outbursts the presence of a large disk was terminated observationally by a small optical rebrightening accompanied by the emergence of the Raman line (Fig. 5 here and Sect. 3.6 in Skopal et al. 2006).

We note that the disk-jet connection in Z And, as summarized in the points above, has some similarities with a hard state of the microquasar GRS 1915+105 as described by Belloni et al. (2000) and later discussed by Livio et al. (2003). For the symbiotic star CH Cyg this similarity was pointed out by Sokoloski \& Kenyon (2003).

\subsection{Variation of jets and disk - a self-induced warping of the disk?}

The asymmetric jet episode in $\mathrm{Z}$ And lasted for less than one month - from the jets launching in 2006 July to the beginning of 2006 August (Fig. 6, Sect. 3.4). The velocity asymmetry in jets was so far revealed only for other objects and at significantly larger timescales of years to decades (e.g. Hirth et al. 1994; Woitas et al. 2002; López-Martín et al. 2003; Namouni 2007). Thus, the very short duration of the asymmetric jets in Z And was probably connected with a disk instability induced during the optical maximum. Qualitatively, the instability could cause different conditions on the accretor's poles for the ejection of jets. Particularly, the anti-correlation of the opening angles to the jet velocities at the initial stage of the jets launching (Fig. 6) could be a possible cause of the observed jet's asymmetry. The larger the jet nozzle is, the lower ejection velocity can be expected to drive the jet flow at the same rate (Eq. (4)). This possibility was already suggested by Hirth et al. (1994) for magnetically driven jets if the opening angles are different due to different pressure gradients. The short-term, $\Delta m \sim 0.06 \mathrm{mag}$, photometric variations represent another type of variability, which developed during the jet ejection (Fig. 3, Sect. 3.1). Their source was associated with the disk (Sect. 3.1). In the previous section we ascribed the evolution in the short-term variability to disruption of the inner parts of the disk.

The origin of both these effects could become better understood by investigating a disk instability due to an increase of the hot component luminosity at the outburst maximum. According to Pringle (1996) irradiation of the disk by the central star can lead an originally planar disk to become twisted and tilted out of the orbital plane. In our case, additional energy liberated during the outburst by thermonuclear burning on the WD surface increases formally the efficiency $\epsilon$ of the accretion process (see Eq. (3.10) in Pringle 1997), which then could lead to radiation-driven warping close to the disk's center even for WD accretors. For Z And, the radiation-induced warping 
occurs at all radii $R \gtrsim 0.6 R_{\odot}$ for the luminosity of $\sim 10^{4} L_{\odot}$ around the optical maximum (e.g. Sokoloski et al. 2006), the mass accretion rate from the wind of $\sim 7 \times 10^{-8} M_{\odot} \mathrm{yr}^{-1}$ (Sect. 4.1) and the WD mass of $0.64 M_{\odot}$ (Sect. 3.6). With respect to the disk radius of $\gtrsim 10 R_{\odot}$ (Sect. 3.1), the warping will act from inner parts of the disk.

However, connections between jets and disk variability in symbiotics have not been studied theoretically in detail yet. Current theories on the warping and wobbling disks were elaborated for different types of objects and are characterized by significantly larger timescales than those suggested by observations of Z And (e.g. Pringle 1996; Livio \& Pringle 1997; Wijers \& Pringle 1999).

\section{Summary}

The main results of this paper can be summarized as follows:

(i) Between 2006 July 19 and 28 our photometric monitoring of the prototypical symbiotic star Z And revealed the highest maximum of its brightness that has ever been recorded by the multicolor photometry ( $U \sim 8.0$, Fig. 1). Around the mid of August the brightness declined to $U \sim 9$ and persisted around this level to 2007 January. During this period, rapid photometric variation ( $\Delta B \sim \Delta V \sim 0.06 \mathrm{mag}$ ) on the timescale of hours developed (Fig. 3).

(ii) The SED models and spectral characteristics from the optical maximum can be explained by a disk-like structure of the hot active object (Sect. 3.3). The short-term photometric variation was produced by the disk (Sect. 3.1).

(iii) During the optical maximum a mass ejection from the active object was indicated photometrically through an increase of the nebular emission (Fig. 2) and spectroscopically by the development of complex absorptions on the blue side of the hydrogen and helium lines and a strong $\mathrm{S}^{+}$jet emission (Fig. 4).

(iv) High velocity satellite components to $\mathrm{H} \alpha$ and $\mathrm{H} \beta$ emission lines developed in the spectrum from the optical maximum. Their presence in the spectrum was transient. Our first and last detection was on 25/07/2006 and 27/12/2006, respectively. Their spectral properties (Table 2) indicated ejection of highly collimated bipolar jets. We summarize their main characteristics as follows:

1. The jets were asymmetrical in their velocities for less than one month from their launching in 2006 July $\left(R V_{\mathrm{S}^{+}} / R V_{\mathrm{S}^{-}}=1.2-1.3\right)$. After this episode they became suddenly symmetrical until the end of their presence (Fig. 6).

2. The jets were collimated within an average opening angle of $6^{\circ} .1$ for the orbital inclination $i=76^{\circ}$ (Sect. 3.5). 
3. The average velocity of jets was $4960 \mathrm{~km} \mathrm{~s}^{-1}$. The ratio $v_{\text {jet }} / v_{\text {escape }} \sim 1$ implies mass of the accreting WD of $\sim 0.64 M_{\odot}$ (Sect. 3.6).

4. The average outflow rate via jets was $\dot{M}_{\text {jet }} \sim 2 \times 10^{-6}\left(R_{\text {jet }} / 1 \mathrm{AU}\right)^{1 / 2} M_{\odot} \mathrm{yr}^{-1}$, during their August-September maximum, which corresponds to the emitting mass in jets, $M_{\text {jet }}^{\mathrm{em}} \sim 6 \times$ $10^{-10}\left(R_{\text {jet }} / 1 \mathrm{AU}\right)^{3 / 2} M_{\odot}($ Sect. 3.8.2).

5. The total mass released by the jets during their detection was approximated to $M_{\text {jet }}^{\text {total }} \approx$ $7.4 \times 10^{-7} M_{\odot}$ (Sect. 3.8.3).

(v) The short duration of the asymmetry in the jet velocities and evolution in the rapid photometric variability resulted from a disruption of the innermost disk. In particular, we pointed out a possibility of the radiation-induced warping, which can occur close to the disk's center due to the additional source of energy from the thermonuclear burning on the WD surface. For Z And parameters, this type of disk instability could occur at all radii $R \gtrsim 0.6 R_{\odot}$ from the accretor (Sect. 4.2).

The authors thank Mario Livio for constructive comments that helped improve the clarity of the paper. Dmitri Bisikalo and Drahomír Chochol are thanked for discussions. Martin Vaňko and Pavel Schalling are thanked for taking some photometric observations and Miroslav Šlechta for initial reduction of the spectra taken at the Ondřejov Observatory. This research was supported in part by a grant of the Slovak Academy of Sciences No. 2/7010/27 and by the Grant Agency of the Czech Republic, Grant No. 205/06/0217 and 205/08/H005. JB acknowledges the support from the Pennsylvania State University and the NSF-NATO grant DGE-0312144 and the Marie Curie international reintegration grant MIRG-CT-2007-200297. The Hobby-Eberly Telescope (HET) is a joint project of the University of Texas at Austin, the Pennsylvania State University, Stanford University, Ludwig-Maximillians-Universität München, and Georg-August-Universität Göttingen. The HET is named in honor of its principal benefactors, William P. Hobby and Robert E. Eberly.

\section{A. Ionization boundary of jets}

Here we calculate the boundary between the ionized and neutral part of the jet for the case that the sources of both the ionizing photons and particles are located at the central star. The boundary is determined by the locus of points, at which the flux of ionizing photons is balanced by the rate of ionization/recombination acts inside the nebula. Thus the equilibrium condition

between the number of ionizing photons $L_{\mathrm{ph}}$, emitted into the fraction of the sphere $\Delta \Omega / 4 \pi$ around the direction of the jet, and the number of recombinations, can be expressed as

$$
L_{\mathrm{ph}} \frac{\Delta \Omega}{4 \pi}=\frac{\Delta \Omega}{4 \pi} \int_{0}^{R_{\mathrm{jet}}} n_{\mathrm{e}} n_{\mathrm{p}} \alpha_{\mathrm{B}}\left(H, T_{\mathrm{e}}\right) 4 \pi r^{2} \mathrm{~d} r
$$




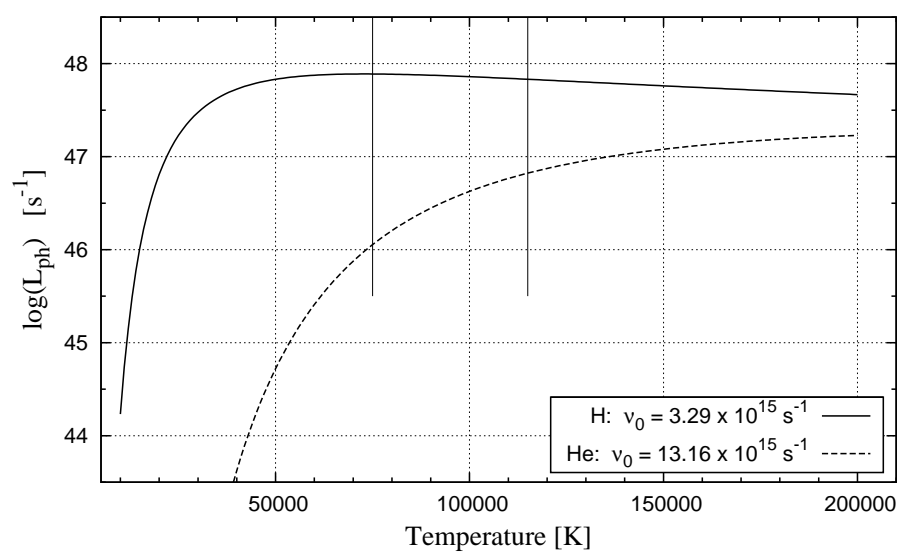

Fig. 9.- Number of photons capable to fully ionize neutral hydrogen and helium as a function of temperature. The photon rates were determined by integrating the Planck curve from the ionization limits $\nu_{0}$ to $\infty$ (see Eq. (11) in Skopal 2001), and scaled to the luminosity of $10000 L_{\odot}$. Solid thin vertical lines limit the range of temperatures derived from fluxes of $\mathrm{H} \beta$ and He II 4686 during the stable stage of jets (Sect. 3.7).

It is assumed that the ionizing photons are emitted by the central source spherically symmetrically, $\alpha_{\mathrm{B}}\left(H, T_{\mathrm{e}}\right)$ stands for the total hydrogenic recombination coefficient in the case $B, n_{\mathrm{e}}$ and $n_{\mathrm{p}}$ are concentrations of electrons and protons and $r$ measures the distance from the central ionizing source along the jet. For the fully ionized hydrogen plasma $\left(n_{\mathrm{e}}=n_{\mathrm{p}}\right)$ with a mean particle concentration, $\bar{n}_{\text {jet }}$, Eq. (A1) can be integrated to give

$$
\frac{L_{\mathrm{ph}}}{4 \pi}=\frac{1}{3} \alpha_{\mathrm{B}}\left(H, T_{\mathrm{e}}\right) \bar{n}_{\text {jet }}^{2} R_{\text {jet }}^{3} .
$$

In the sense of this relation, the radius of the visible part of the jet, $R_{\text {jet }}$, represents that of the Strömgren sphere.

\section{B. Number of ionizing photons}

To determine the total number of photons, $L_{\mathrm{ph}}$, capable to ionize neutral hydrogen and helium, emitted by the star of temperature $T$, we integrated the Plank curve from the corresponding ionization limits (i.e. $13.598 \mathrm{eV}, \nu_{0}=3.29 \times 10^{15} \mathrm{~s}^{-1}$ and $54.416 \mathrm{eV}, \nu_{0}=13.16 \times 10^{15} \mathrm{~s}^{-1}$ for hydrogen and helium, respectively) to $\infty$. Figure 9 shows the $L_{\mathrm{ph}}$ quantity as a function of the temperature for a luminosity of the ionizing source of $10000 L_{\odot}$. It is worth to note that the function $L_{\mathrm{ph}}(T, H)$ is slightly decreasing from $\sim 73000 \mathrm{~K}$ to higher temperatures, in spite that the total energy beyond the ionization limit increases. Therefore, irrespective to a large temperature changes between 50000 and $125000 \mathrm{~K}$, the number of the hydrogen ionizing photons varies only within $15 \%$ of the maximum value at $73000 \mathrm{~K}$ at a given luminosity (see Fig. 9). 


\section{REFERENCES}

Belloni, T., Klein-Wolt, M., Méndez, M., van der Klis, M., \& van Paradijs, J. 2000, A\&A, 355, 271

Bessell, M. S. 1983, PASP, 95, 480

Bisikalo, D. V., Boyarchuk, A. A., Kilpio, E. Yu., Tomov, N. A., Tomova, M. T. 2006, Astron. Rep., 80, 722

Brocksopp, C., Sokoloski, J. L., Kaiser, C., Richards, A.M., Muxlow, T.W.B., \& Seymour, N. 2004, MNRAS, 347,430

Burmeister, M., \& Leedjärv, L. 2007, A\&A, 461, L5

Crocker, M. M., Davis R. J., Eyres S. P. S., Bode M. F., Taylor A. R., Skopal A., Kenny H. T., 2001, MNRAS, 326, 781

Fekel, F. C., Hinkle, K. H., Joyce, R. R., \& Skrutskie, M. F. 2000, AJ, 120, 3255

Fernández-Castro, T., González-Riestra, R., Cassatella, A., Taylor, A. R., \& Seaquist E. R. 1995, ApJ, 442, 366

Formiggini, L. \& Leibowitz, E. M. 1994, A\&A, 292, 534

Galloway, D. K., \& Sokoloski, J. L. 2004, ApJL, 613, 61

Gromadzki, M., Mikolajewski, M. Tomov, T., Bellas-Velidis, I., Dapergolas, A., \& Galan, C. 2006, Acta Astron., 56, 97

Gurzadyan, G. A. 1997, The Physics and Dynamics of Planetary Nebulae, (Berlin: Springer)

Hirth, G. A., Mundt, R., Solf, J., \& Ray, T. P. 1994, ApJ, 427, L99

Horn, J., Kubát, J., Harmanec, P., et al. 1996, A\&A, 309, 521

Iijima, T. 1981, in NATO Advanced Study Institute 69, Photometric and Spectroscopic Binary Systems, 517

Izumiura, H. 1999, in Observational Astrophysics in Asia and its Future, ed. P. S. Chen, (Yunnan Observatory: Chinese Academy of Sciences) p.77

Karovska, M., Carilli, Ch. L., Raymong, J. C., \& Mattei, J. A. 2007, ApJ, 661, 1048

Kellogg, E., Anderson, C., Korreck, K., DePasquale, J., Nichols, J., Sokoloski, J. L., Krauss, M., Pedelty, J. 2007, ApJ, 664, 1079

Lamers, H. J. G. L. M., \& Cassinelli, L. P. 1999, Introduction to Stellar Winds (Cambridge: Cambridge Univ. Press)

Livio, M. 1997, in ASP Conf. Ser. 121, Accretion Phenomena and Related Outflows, ed. D. T. Wickramashinge, G. V. Bicknell, \& L. Ferrario, (San Francisco, CA: ASP), 845

Livio, M. 2004, Baltic Astron., 13, 273

Livio, M., \& Pringle, J. E. 1997, ApJ, 486, 835

Livio, M., Pringle, J. E., \& King, A. R. 2003, ApJ, 593, 184

Lizano, S., Heiles, C., Rodriguez, L. F., Koo, B.-C., Shu, F. H., Hasegawa, T., Hayashi, S., \& Mirabel, I. F. 1988, ApJ, 328, 763

López-Martín, L., Cabrit, S., \& Dougados, C. 2003, A\&A, 405, L1 
Mikolajewska, J., \& Kenyon, S. J. 1992, MNRAS, 256, 177

Mikolajewska, J., \& Kenyon, S. J. 1996, AJ, 112, 1659

Mürset, U., \& Nussbaumer, H. 1994, A\&A, 282, 586

Nagae, T., Oka, K., Matsuda, T., et al. 2004, A\&A, 419, 335

Namouni, F. 2007, ApJ, 659, 1505

Nussbaumer, H., \& Vogel, M. 1987, A\&A, 182, 51

Osterbrock, D. E. 1989, Astrophysics of Gaseous Nebulae and Active Galactic Nuclei (San Francisco, CA: Freeman)

Parimucha, Š., \& Vaňko, M. 2005, CoSka, 35, 35

Pringle, J. E. 1993, in Astrophysical Jets, ed. D. Burgerela, M. Livio \& C. O’Dea (Cambridge: Cambridge Univ. Press), 1

Pringle, J. E. 1996, MNRAS, 281, 357

Pringle, J. E. 1997, MNRAS, 292, 136

Ramsey, L. W., et al. 1998, in Proc. SPIE, 3352, 34

Schmid, H. M., \& Schild, H. 1997, A\&A, 327, 219

Schmid, H. M., Krautter, J., Appenzeller, I., et al. 1999, A\&A, 348, 950

Schmid, H. M., Kaufer, A., Camenzind, M., et al. 2001, A\&A, 377, 206

Shahbaz, T., Livio, M., Southwell, K. A., \& Charles, P. A. 1997, ApJ, 484, L59

Shapiro, S. L., \& Teukolsky, S. A. 1983, Black Holes, White Dwarfs, and Neutron Stars (New York: Wiley)

Skopal, A. 1998, A\&A, 338, 599

Skopal, A. 2001, A\&A, 366, 157

Skopal, A. 2003, A\&A, 401, L17

Skopal, A. 2005, A\&A, 440, 995

Skopal, A. 2006, A\&A, 457, 1003

Skopal, A. 2007, New Astron., 12, 597

Skopal, A., Bode, M.F., Crocker, M. M., Drechsel, H., Eyres, S. P. S., \& Komžík, R. 2002, MNRAS, 335, 1109

Skopal, A., Pribulla, T., Vaňko, M., Velič, Z., Semkov, E., Wolf, M., \& Jones, A. 2004, CoSka, 34, 45

Skopal, A., \& Pribulla, T. 2006, Astron. Tel., 882

Skopal, A., \& Wolf, M. 2006, Astron. Tel., 930

Skopal, A., Vittone, A. A., Errico, L., Otsuka, M., Tamura, S., Wolf, M., \& Elkin, V. G. 2006, A\&A, 453, 279

Soker, N. \& Regev, O. 2003, A\&A, 406, 603 
Soker, N. \& Lasota, J.-P. 2004, A\&A, 422, 1039

Sokoloski, J. L., \& Bildsten, L. 1999, ApJ, 517, 919

Sokoloski, J. L., \& Kenyon, S. J. 2003, ApJ, 584, 1021

Sokoloski, J. L., Kenyon, S. J., Brocksopp, C., Kaiser, R. C., \& Kellogg, E. M. 2004, RevMexAA, 20, 35

Sokoloski, J. L., Kenyon, S. J., Espey, B. R., et al. 2006, ApJ, 636, 1002

Stute, M., \& Camenzind, M. 2005, A\&A, 432, L17

Stute, M., \& Sahai, R. 2007, ApJ, 665, 698

Škoda, P. 1996, in ASP Conf. Ser. 101, Astronomical Data Analysis Software and Systems V, ed. G. H. Jacoby, \& J. Barnes, (San Francisco, CA: ASP), 187

Taylor, A. R., Seaquist, E. R., \& Mattei, J. A. 1986, Nature, 319, 38

Tomov, T., Munari, U., \& Marrese, P. M. 2000, A\&A, 354, L25

Tomov, N. A., Taranova, O. G., \& Tomova, M. T. 2003, A\&A, 401, 669

Tomov, N. A., Tomova, M. T., \& Bisikalo, D. V. 2007, MNRAS, 376, L16

Tomov, N. A., Tomova, M. T., \& Bisikalo, D. V. 2008, MNRAS, 389, 829

Tull, R. G. 1998, in Proc. SPIE, 3355, Optical Astronomical Instrumentation, ed. S. D'odorico (Bellingham: WA: SPIE), 387

Wijers, R. A. M. J., \& Pringle, J. E. 1999, MNRAS, 308, 207

Woitas, J., Ray, T. P., Bacciotti, F., Davis, C. J., \& Eislöffel, J. 2002, ApJ, 580, 336 